\title{
Naturalness of light neutralino dark matter in pMSSM after LHC, XENON100 and Planck data
}

\author{
Céline Bœhm, ${ }^{a, b}$ P.S. Bhupal Dev, ${ }^{c}$ Anupam Mazumdar ${ }^{d, e}$ and Ernestas Pukartas ${ }^{d}$ \\ ${ }^{a}$ Institute for Particle Physics Phenomenology, University of Durham, Durham DH1 3LE, U.K. \\ ${ }^{b}$ LAPTh, U. de Savoie, CNRS, BP 110, 74941 Annecy-Le-Vieux, France \\ ${ }^{c}$ Consortium for Fundamental Physics, School of Physics and Astronomy, \\ University of Manchester, Manchester M13 9PL, U.K. \\ ${ }^{d}$ Consortium for Fundamental Physics, Lancaster University, Lancaster LA1 4YB, U.K. \\ ${ }^{e}$ Niels Bohr Institute, Copenhagen University, Blegdamsvej-17, Copenhagen, Denmark \\ E-mail: c.m.boehm@durham.ac.uk, Bhupal.Dev@hep.manchester.ac.uk, \\ a.mazumdar@lancaster.ac.uk, e.pukartas@lancaster.ac.uk
}

ABSTRACT: We examine the possibility of a light (below $46 \mathrm{GeV}$ ) neutralino dark matter (DM) candidate within the 19-parameter phenomenological Minimal Supersymmetric Standard Model (pMSSM) in the light of various recent experimental results, especially from the LHC, XENON100, and Planck. We also study the extent of electroweak fine-tuning for such a light neutralino scenario in view of the null results from the searches for supersymmetry so far. Using a Markov Chain Monte Carlo likelihood analysis of the full pMSSM parameter space, we find that a neutralino DM with mass $\gtrsim 10 \mathrm{GeV}$ can in principle still satisfy all the existing constraints. Our light neutralino solutions can be broadly divided into two regions: (i) The solutions in the $10-30 \mathrm{GeV}$ neutralino mass range are highly finetuned and require the existence of light selectrons (below $100 \mathrm{GeV}$ ) in order to satisfy the observed DM relic density. We note that these are not yet conclusively ruled out by the existing LEP/LHC results, and a dedicated analysis valid for a non-unified gaugino mass spectrum is required to exclude this possibility. (ii) The solutions with low fine-tuning are mainly in the $30-46 \mathrm{GeV}$ neutralino mass range. However, a major portion of it is already ruled out by the latest XENON100 upper limits on its spin-independent direct detection cross section, and the rest of the allowed points are within the XENON1T projected limit. Thus, we show that the allowed MSSM parameter space for a light neutralino DM below the LEP limit of $46 \mathrm{GeV}$, possible in supersymmetric models without gaugino mass unification, could be completely accessible in near future. This might be useful in view of the recent claims for positive hints of a DM signal in some direct detection experiments.

KEYWORDS: Supersymmetry Phenomenology

ARXIV EPRINT: 1303.5386 


\section{Contents}

1 Introduction 1

2 Electroweak fine-tuning $\quad 4$

3 Parameters and constraints $\quad 6$

4 Results 12

5 Conclusion 18

\section{Introduction}

Low-scale Supersymmetry (SUSY) (see e.g., [1, 2]) is one of the most attractive candidates for New Physics beyond the Standard Model (SM). Apart from providing successful gauge coupling unification and a solution to the gauge hierarchy problem, it offers a natural candidate for Dark Matter (DM) in our Universe in the form of the lightest supersymmetric particle (LSP). In the $R$-parity conserving Minimal Supersymmetric Standard Model (MSSM), the lightest neutralino $\left(\widetilde{\chi}_{1}^{0}\right)$ is one of the most viable Weakly Interacting Massive Particle (WIMP) DM candidates (for a review, see e.g., [3, 4]). It can explain the observed DM relic density, while predicting experimentally accessible direct and indirect detection rates, over a wide range of supersymmetric model parameters, some of which are already getting constrained from the ongoing direct searches for the supersymmetric particles at the Large Hadron Collider (LHC), in combination with other low-energy data (for a review, see e.g., [5]).

The recent hints of positive signals in three DM direct detection experiments, namely, DAMA [6, 7], CoGeNT [8] and CRESST-II [9], have generated a lot of interest in light WIMP candidates in the $5-50 \mathrm{GeV}$ mass range. This interpretation is however challenged by the null results from various other direct detection experiments, most notably the latest XENON100 results [10], which provide the most stringent upper limits on the spinindependent WIMP-nucleon scattering cross section for $m_{\chi}>7 \mathrm{GeV} .{ }^{1}$ Nonetheless, due to the relatively poor sensitivity of the XENON100 experiment in the very low WIMP mass regime, it is believed that an agreement between the positive and null sets of experimental results could be possible, if at all, only in this low mass region. Hence, it might be worthwhile examining the allowed MSSM parameter space to see if there exists a lower bound on the lightest neutralino mass irrespective of the direct detection results.

\footnotetext{
${ }^{1}$ More recently, the TEXONO experiment [11] has achieved slightly better sensitivity than XENON100 for $m_{\chi}<7 \mathrm{GeV}$.
} 
The neutralino mass eigenstates in the MSSM result from mixing of the neutral bino $(\widetilde{B})$, wino $\left(\widetilde{W}^{0}\right)$ and higgsinos $\left(\widetilde{H}_{d}^{0}, \widetilde{H}_{u}^{0}\right)$. This mixing is determined by the MSSM $\tan \beta$ parameter and the bino, wino and higgsino mass parameters $M_{1}, M_{2}$ and $\mu$, respectively. In the SUSY models with gaugino mass unification at the Grand Unified Theory (GUT) scale, a relation between the bino and wino mass follows at the electroweak scale: $M_{1}=\frac{5}{3} \tan ^{2} \theta_{W} M_{2} \approx 0.5 M_{2}$ (see e.g., [12]) which, after mixing, translates into a charginoneutralino mass relation. Therefore, a lower limit on the lightest neutralino $\left(\widetilde{\chi}_{1}^{0}\right)$ mass of about $46 \mathrm{GeV}$ can be derived for these models from the Large Electron Positron (LEP) chargino mass limit $[13,14]$, whereas in the constrained MSSM (cMSSM) [15] with both gaugino and sfermion mass unification, this limit increases to well above $100 \mathrm{GeV}$ from the strong constraints set by the recent LHC data [5].

On the other hand, in a generic MSSM scenario without the assumption of gaugino mass unification, there is no general lower limit on the lightest neutralino mass [16]. The LEP limit on the invisible decay width of the SM $Z$ boson applies to light neutralinos with a mass below $m_{Z} / 2=45.6 \mathrm{GeV}$, but it depends on the $Z \widetilde{\chi}_{1}^{0} \overline{\widetilde{\chi}}_{1}^{0}$ coupling which could be small or even zero, depending on the higgsino component of the neutralino. In such a case, light neutralinos are mainly constrained by the DM relic density measurement as well as by the collider and flavor constraints on the SUSY parameter space. Assuming that the lightest neutralino is non-relativistic and provides the entire cold DM content of the Universe, while satisfying the LEP bounds on chargino and stau masses, ref. [17] obtained a lower bound of $m_{\widetilde{\chi}_{1}^{0}} \gtrsim 18 \mathrm{GeV}$. This was relaxed to about $6 \mathrm{GeV}$ without violating the LEP bounds and flavor sector constraints in SUSY models with a pseudo-scalar Higgs boson $(A)$ mass $m_{A}<200 \mathrm{GeV}$ and a large $\tan \beta[18,19]$. This was even further lowered to about $3 \mathrm{GeV}$ by allowing explicit $C P$ violation in the MSSM Higgs sector [20].

Meanwhile, several new experimental results have been obtained at the LHC: (i) A new Higgs-like neutral scalar particle has been discovered [21, 22] with mass around $125 \mathrm{GeV}$ which falls squarely within the MSSM-predicted range for the lightest $C P$-even neutral Higgs mass: $m_{h} \in[115,135] \mathrm{GeV}$ (see e.g., [23]); (ii) The rare decay $B_{s}^{0} \rightarrow \mu^{+} \mu^{-}$was observed with a branching fraction of $\mathcal{B}\left(B_{s}^{0} \rightarrow \mu^{+} \mu^{-}\right)=\left(3.2_{-1.2}^{+1.5}\right) \times 10^{-9}$ [24] which is in agreement with the SM expectation, $\mathcal{B}\left(B_{s}^{0} \rightarrow \mu^{+} \mu^{-}\right)_{\mathrm{SM}}=(3.23 \pm 0.27) \times 10^{-9}[25]$; (iii) The lack of a SUSY signal at the $\sqrt{s}=7$ and $8 \mathrm{TeV}$ LHC has pushed the lower limits on the squark and gluino masses to about $1 \mathrm{TeV}$ and beyond [26, 27]; (iv) Updated bounds have been obtained for the MSSM Higgs sector [28-30]. All these new results have profound implications for a light neutralino scenario within the MSSM, and some of these aspects have already been investigated in a number of recent studies [31-45]. The general conclusion is that light neutralino DM candidates with mass below about $15 \mathrm{GeV}$ are severely constrained in generic MSSM scenarios (without gaugino mass unification).

Another important issue to be addressed in the light of the recent LHC results is the apparent "little hierarchy problem", i.e., how does a multi-TeV SUSY particle spectrum conspire to give a weak-scale $Z$-boson mass and also a Higgs boson mass of $125 \mathrm{GeV}$ ? One way of analyzing this issue quantitatively is to evaluate the measure of electroweak finetuning (EWFT) by examining the minimization condition in the MSSM Higgs potential which determines the $Z$-boson mass $[46,47]$. It is well-known that radiative corrections 
must play a crucial role in determining the allowed SUSY parameter space necessary to generate a $125 \mathrm{GeV}$ Higgs boson mass, much larger than its tree-level prediction of $m_{h} \leq m_{Z}$. This in general can lead to a large fine-tuning. In addition to this, the requirement of a light neutralino DM will pose a challenge for any MSSM scenario, the severity of which is however strongly model-dependent. The naturalness of various SUSY models with a neutralino LSP has been analyzed in the literature (for an incomplete list, see [42, 48-61], and references therein).

In this paper, we perform a dedicated study focusing on the naturalness of a light neutralino, and also examining how light the neutralino could be, after taking into account all the existing theoretical and experimental constraints. To perform such an analysis in the full 124-parameter MSSM is quite unrealistic, and hence, we need to make some wellmotivated assumptions in order to reduce the number of parameters to a manageable level. Most of the earlier studies on SUSY focused on the cMSSM having only 5 parameters, assuming certain boundary conditions at the GUT-scale. However, in view of the latest null results from SUSY searches at the $\sqrt{s}=8 \mathrm{TeV}$ LHC, the cMSSM seems too restrictive for low-scale SUSY phenomenology as the allowed cMSSM parameter space accessible to the $\sqrt{s}=14 \mathrm{TeV}$ LHC is rapidly shrinking (for the latest global status, see e.g., ref. [6264]). Therefore, in this paper we choose not to make any assumptions at the high scale, and focus only on the low-scale MSSM parameter space from a phenomenological point of view. More precisely, we consider a $C P$-conserving MSSM (i.e., with no new $C P$ phases) with Minimal Flavor Violation [65] and with first two generations of sfermions degenerate. This is widely known as the phenomenological MSSM (pMSSM) [66] (also known as 'SUSY without prejudice' [67]) with 19 free parameters at the electroweak scale. We also study the level of fine-tuning for the light neutralino scenario in this context.

In order to efficiently explore the 19-dimensional pMSSM parameter space, we perform a Markov Chain Monte Carlo (MCMC) likelihood analysis (for a review, see e.g., [68]), with the priors chosen to focus on a light neutralino scenario with mass below the conservative LEP lower limit of $46 \mathrm{GeV}$. We include in our analysis the latest experimental results for SUSY searches from the LHC [26, 27] which now supersede the Tevatron results [69, 70], in addition to the existing LEP limits $[13,14]$, wherever applicable. We also include the latest astrophysical/cosmological constraints for a WIMP DM from the 9-year WMAP data [71] as well as the very recently released Planck data [72]. We further examine the allowed parameter space in the light of various recent results for DM direct detection, most notably the XENON100 limits [10], as well as the indirect detection results from Fermi-LAT data [73].

We find that a light neutralino DM with mass as low as $10 \mathrm{GeV}$ is still allowed in the pMSSM, while satisfying all the existing experimental constraints provided we only take the model-independent analysis results from LEP. (Including the LEP limits strictly applicable to gaugino-mass unification models allows only the solutions with $m_{\widetilde{\chi}_{1}^{0}}>30 \mathrm{GeV}$, in agreement with previous results $[33,34]$.) However, such neutralinos which are required to be mostly bino-like are severely fine-tuned and require the existence of light sleptons below $100 \mathrm{GeV}$ in order to provide an efficient annihilation channel to reduce the bino relic density to be consistent with the observed limit. A dedicated analysis of the LEP data in the context of a pMSSM scenario could eliminate this region completely. On the other hand, low 
fine-tuning regions can be obtained around $m_{\widetilde{\chi}_{1}^{0}}=45 \mathrm{GeV}$ where the resonant annihilation via the $s$-channel $Z$-exchange is possible for the neutralino with a non-negligible higgsino component. However, such regions are mostly excluded by the recent XENON100 limits on the spin-independent DM-nucleon scattering cross section, and the remaining such points are well within the reach of the XENON1T [74] and LUX [75] projected limits.

Our paper is organized as follows: in section 2, we briefly discuss the electroweak finetuning measure. In section 3, we list all the pMSSM parameters and their scan ranges, and also summarize all the relevant experimental constraints used in our numerical analysis. In section 4, we present our scan results and discuss their implications for a light neutralino DM. Finally, our conclusions are given in section 5 .

\section{Electroweak fine-tuning}

To quantify the amount of fine-tuning in the electroweak symmetry breaking (EWSB) sector of the MSSM, it is sufficient for us to analyze the tree-level MSSM scalar potential. Since it allows only charge-conserving vacua, we only have to minimize the scalar potential for the neutral scalar fields [1]:

$$
\begin{aligned}
V= & \left(m_{H_{u}}^{2}+\mu^{2}\right)\left|H_{u}^{0}\right|^{2}+\left(m_{H_{d}}^{2}+\mu^{2}\right)\left|H_{d}^{0}\right|^{2} \\
& -B \mu\left(H_{u}^{0} H_{d}^{0}+\text { H.c. }\right)+\frac{1}{8}\left(g^{2}+g^{\prime 2}\right)\left(\left|H_{u}^{0}\right|^{2}-\left|H_{d}^{0}\right|^{2}\right)^{2},
\end{aligned}
$$

where $\mu$ is the SUSY-preserving bilinear Higgs superpotential parameter, $m_{H_{u, d}}$ and $B$ are the soft scalar masses and the bilinear coupling in the SUSY-breaking sector respectively, and $g, g^{\prime}$ the $\mathrm{SU}(2)_{L}$ and $\mathrm{U}(1)_{Y}$ gauge couplings, respectively. After minimization, we obtain the well-known relation for the $Z$-boson mass:

$$
\frac{m_{Z}^{2}}{2}=\frac{m_{H_{d}}^{2}-m_{H_{u}}^{2} \tan ^{2} \beta}{\tan ^{2} \beta-1}-\mu^{2}
$$

where $\tan \beta=v_{u} / v_{d}$ is the ratio of the vacuum expectation values (vevs) of the two Higgs doublet fields $H_{u}$ and $H_{d}$ respectively, It is clear from eq. (2.2) that a cancellation of the terms on the right hand side (RHS) is required in order to obtain the measured value of $m_{Z}=91.2 \mathrm{GeV}[5]$ especially if the mass parameters on the RHS are orders of magnitude larger than the weak scale which indeed seems to be the case, given the current experimental status of the direct SUSY searches [26, 27]. Thus naively speaking, the weak scale value of $|\mu|$ can be used as a measure of fine-tuning in the MSSM.

A more sophisticated way to quantify the degree of EWFT is by using logderivatives [47]:

$$
\Delta p_{i}=\left|\frac{\partial \ln m_{Z}^{2}\left(p_{i}\right)}{\partial \ln p_{i}}\right|=\left|\frac{p_{i}}{m_{Z}^{2}} \frac{\partial m_{Z}^{2}}{\partial p_{i}}\right|
$$

where $p_{i}$ 's are the parameters that determine the observable $Z$-mass at tree-level. From eq. (2.2), we have $p_{i}=\left\{\mu^{2}, b, m_{H_{u}}, m_{H_{d}}\right\}$ (with $b \equiv B \mu$ ), and the total measure of the EWFT is defined as

$$
\Delta_{\text {tot }}=\sqrt{\left(\Delta \mu^{2}\right)^{2}+(\Delta b)^{2}+\left(\Delta m_{H_{u}}^{2}\right)^{2}+\left(\Delta m_{H_{d}}^{2}\right)^{2}}
$$


with the individual $\Delta p_{i}$ 's given by [76]

$$
\begin{aligned}
\Delta \mu^{2} & =\frac{4 \mu^{2}}{m_{Z}^{2}}\left(1+\frac{m_{A}^{2}+m_{Z}^{2}}{m_{A}^{2}} \tan ^{2} 2 \beta\right), \\
\Delta b & =\left(1+\frac{m_{A}^{2}}{m_{Z}^{2}}\right) \tan ^{2} 2 \beta, \\
\Delta m_{H_{u}}^{2} & =\left|\frac{1}{2} \cos 2 \beta+\frac{m_{A}^{2}}{m_{Z}^{2}} \cos ^{2} \beta-\frac{\mu^{2}}{m_{Z}^{2}}\right|\left(1-\frac{1}{\cos 2 \beta}+\frac{m_{A}^{2}+m_{Z}^{2}}{m_{A}^{2}} \tan ^{2} 2 \beta\right), \\
\Delta m_{H_{d}}^{2} & =\left|-\frac{1}{2} \cos 2 \beta+\frac{m_{A}^{2}}{m_{Z}^{2}} \sin ^{2} \beta-\frac{\mu^{2}}{m_{Z}^{2}}\right|\left(1+\frac{1}{\cos 2 \beta}+\frac{m_{A}^{2}+m_{Z}^{2}}{m_{A}^{2}} \tan ^{2} 2 \beta\right) .
\end{aligned}
$$

Here $m_{A}^{2}=B \mu(\tan \beta+\cot \beta)$ is the MSSM pseudo-scalar Higgs mass, and we have assumed $\tan \beta>0$. Values of $\Delta_{\text {tot }} \gg 1$ indicate significant fine-tuning. Note that in the decoupling limit with $m_{A} \gg m_{Z}$ and with large $\tan \beta$, the quantities $\Delta m_{H_{u}}^{2}$ and $\Delta m_{H_{d}}^{2}$ in eq. (2.5) are small, and

$$
\Delta \mu^{2} \simeq \frac{4 \mu^{2}}{m_{Z}^{2}}, \quad \Delta b \simeq \frac{4 m_{A}^{2}}{m_{Z}^{2} \tan ^{2} \beta} .
$$

Thus in the limit of large $\tan \beta$, we recover the naive result that fine-tuning increases with increasing $|\mu|$.

We should note here that including loop corrections to eq. (2.2), one finds the largest contribution to be coming from the (s)top loop which feeds into the soft mass of the Higgs [12]:

$$
\delta m_{H_{u}}^{2}=-\frac{3 y_{t}^{2}}{8 \pi^{2}}\left(m_{\widetilde{t}_{L}}^{2}+m_{\widetilde{t}_{R}}^{2}+\left|A_{t}\right|^{2}\right) \log \left(\frac{\Lambda}{m_{\widetilde{t}}}\right),
$$

where $y_{t}$ is the top-quark Yukawa coupling, $A_{t}$ is the third-generation $A$-term in the SUSYbreaking sector, and $\Lambda$ is some high scale where the stop masses $m_{\widetilde{t}_{L, R}}$ are generated from the SUSY-breaking mechanism. Even for a low-scale SUSY-breaking scenario (such as gauge mediation), this requires a fine-tuning of at least a few percent in order to get the observed $Z$-mass and it becomes worse for heavy stop masses [77]. A heavy physical stop mass $m_{\tilde{t}} \gtrsim 500 \mathrm{GeV}$ is anyway required to provide large enough radiative corrections to the light $C P$-even Higgs mass to raise it to the vicinity of $125 \mathrm{GeV}$ from its tree-level value $\leq m_{Z}[12]$ :

$$
\delta m_{h^{0}}^{2}=\frac{3 m_{t}^{4}}{16 \pi^{2} v^{2}}\left[\log \left(\frac{m_{\widetilde{t}_{L}}^{2} m_{\widetilde{t}_{R}}^{2}}{m_{t}^{4}}\right)+\frac{X_{t}^{2}}{m_{\widetilde{t}_{L}} m_{\widetilde{t}_{R}}}\left(1-\frac{X_{t}^{2}}{12 m_{\widetilde{t}_{L}} m_{\widetilde{t}_{R}}}\right)\right],
$$

where $X_{t}=A_{t}-\mu \cot \beta$ is the stop mixing parameter. A fine-tuning measure $\Delta_{m_{h}}$ for the Higgs mass can be defined analogous to eq. (2.3), and for a particular choice of some of the SUSY parameters, it was found that $\Delta_{m_{h}}>75$ (100) in order to achieve a Higgs mass of 124 (126) GeV and the corresponding stop mass is always heavier than 300 (500) GeV [78]. The fine-tuning due to eqs. (2.7) and (2.8) could in principle be added in quadratures to our "total" fine-tuning parameter defined by eq. (2.4), but since their specific values are scale-dependent ${ }^{2}$ and adds to some arbitrariness in its definition, we do not include them in our analysis.

\footnotetext{
${ }^{2}$ The SUSY-scale by convention is usually taken to be the geometric mean of the two stop masses.
} 


\begin{tabular}{|c|c|c|}
\hline Parameter & Description & Prior Range \\
\hline $\tan \beta$ & Ratio of the scalar doublet vevs & {$[1,60]$} \\
$\mu$ & Higgs-Higgsino mass parameter & {$[-3,3] \mathrm{TeV}$} \\
$M_{A}$ & Pseudo-scalar Higgs mass & {$[0.3,3] \mathrm{TeV}$} \\
$M_{1}$ & Bino mass & {$[-0.5,0.5] \mathrm{TeV}$} \\
$M_{2}$ & Wino mass & {$[-1,1] \mathrm{TeV}$} \\
$M_{3}$ & Gluino mass & {$[0.8,3] \mathrm{TeV}$} \\
$m_{\widetilde{q}_{L}}$ & First/second generation $Q_{L}$ squark & {$[0,3] \mathrm{TeV}$} \\
$m_{\widetilde{u}_{R}}$ & First/second generation $U_{R}$ squark & {$[0,3] \mathrm{TeV}$} \\
$m_{\widetilde{d}_{R}}$ & First/second generation $D_{R}$ squark & {$[0,3] \mathrm{TeV}$} \\
$m_{\widetilde{\ell}_{L}}$ & First/second generation $L_{L}$ slepton & {$[0,3] \mathrm{TeV}$} \\
$m_{\widetilde{e}_{R}}$ & First/second generation $E_{R}$ slepton & {$[0,3] \mathrm{TeV}$} \\
$m_{\widetilde{Q}_{3 L}}$ & Third generation $Q_{L}$ squark & {$[0,3] \mathrm{TeV}$} \\
$m_{\widetilde{t}_{R}}$ & Third generation $U_{R}$ squark & {$[0,3] \mathrm{TeV}$} \\
$m_{\widetilde{b}_{R}}$ & Third generation $D_{R}$ squark & {$[0,3] \mathrm{TeV}$} \\
$m_{\widetilde{L}_{3 L}}$ & Third generation $L_{L}$ slepton & {$[0,3] \mathrm{TeV}$} \\
$m_{\widetilde{\tau}_{R}}$ & Third generation $E_{R}$ slepton & {$[0,3] \mathrm{TeV}$} \\
$A_{t}$ & Trilinear coupling for top quark & {$[-10,10] \mathrm{TeV}$} \\
$A_{b}$ & Trilinear coupling for bottom quark & {$[-10,10] \mathrm{TeV}$} \\
$A_{\tau}$ & Trilinear coupling for $\tau$-lepton & {$[-10,10] \mathrm{TeV}$} \\
\hline
\end{tabular}

Table 1. The pMSSM parameters and their range of values used in our numerical analysis.

\section{Parameters and constraints}

We consider the pMSSM with 19 free parameters at the SUSY scale, as shown in table 1. We further assume that the lightest neutralino is the LSP, and our goal is to examine how light and natural the neutralino could be while satisfying all the existing theoretical and experimental constraints on the MSSM parameter space. In particular, we focus on the lightest neutralino masses below the LEP limit of $46 \mathrm{GeV}[13,14]$ which is strictly valid assuming gaugino mass unification at the GUT-scale. We perform a numerical scan over the 19-dimensional pMSSM parameter space using a MCMC-based likelihood analysis for a light neutralino with the prior ranges given in table 1 . These particular ranges are chosen in order to economize the scan time and to focus only on the SUSY parameter space not yet disfavored by the combined direct search results from the LEP [13, 14], Tevatron $[69,70]$ and LHC [26, 27], as discussed later in this section. For the SM parameters $\alpha_{s}\left(m_{Z}\right), \alpha_{\mathrm{em}}\left(m_{Z}\right), m_{W}, m_{t}$ and $m_{b}$, we use the standard values as given in ref. [5].

Apart from the direct collider constraints on the sparticle masses, there exist various theoretical and experimental constraints which must be imposed on the pMSSM parameter 
space in our analysis. As a standard theoretical requirement, our sparticle spectrum for each allowed point in the parameter space must be tachyon-free and should not lead to color- and charge-breaking minima in the scalar potential [79]. We also require that the scalar potential is bounded from below and is consistent with electroweak symmetry breaking. From the radiative electroweak symmetry breaking arguments, we can restrict the $\tan \beta$ parameter to be roughly between $1-60$, as given in table 1 .

The various experimental constraints from direct collider searches, Higgs and flavor sectors, and astrophysical/cosmological data used in our analysis are summarized below.

\section{Invisible $Z$-decay width}

The precise measurement of the $Z$-boson decay width at LEP: $\Gamma_{Z}^{\text {tot }}=2495.2 \pm 2.3 \mathrm{MeV}[80]$ puts severe constraints on light neutralinos and charginos with mass $<m_{Z} / 2$. From the LEP measurements of the invisible decay width of the $Z$-boson: $\Gamma_{Z}^{\text {inv }}=(499.0 \pm$ 1.5) $\mathrm{MeV}$ [80], the parameter space for the lightest neutralino in our case is restricted to mostly gaugino-like scenarios $\left(|\mu| \gg M_{1,2}\right)$ since the neutralino coupling to $Z$ is only via its higgsino component. The allowed fraction of the higgsino component for a given neutralino mass can be calculated using the following expression for the partial decay width of the $Z$-boson to neutralinos [81]:

$$
\frac{\Gamma\left(Z \rightarrow \widetilde{\chi}_{1}^{0} \widetilde{\chi}_{1}^{0}\right)}{\Gamma(Z \rightarrow \nu \bar{\nu})}=2\left(1-\frac{4 m_{\widetilde{\chi}_{1}^{0}}^{2}}{m_{Z}^{2}}\right)^{1 / 2}\left[\left(1-\frac{m_{\widetilde{\chi}_{1}^{0}}^{2}}{m_{Z}^{2}}\right)\left[\left(O_{11}^{L}\right)^{2}+\left(O_{11}^{R}\right)^{2}\right]+\frac{6 m_{\widetilde{\chi}_{1}^{0}}^{2}}{m_{Z}^{2}} O_{11}^{L} O_{11}^{R}\right]
$$

where $\Gamma(Z \rightarrow \nu \bar{\nu})=(501.62 \pm 0.10) \mathrm{MeV}$ is the SM contribution to its invisible decay width (for 3 neutrino species). The components $O_{i j}^{L, R}$ are defined as

$$
O_{i j}^{L}=-\frac{1}{2} N_{i 3} N_{j 3}^{*}+\frac{1}{2} N_{i 4} N_{j 4}^{*}, \quad O_{i j}^{R}=-\left(O_{i j}^{L}\right)^{*}
$$

with $N_{i j}$ measuring the gaugino-Higgsino mixing:

$$
\widetilde{\chi}_{i}^{0}=\sum_{k=1}^{4} N_{i k} \widetilde{\psi}_{k}^{0}, \quad \text { where } \widetilde{\psi}^{0}=\left(\widetilde{B}, \widetilde{W}^{0}, \widetilde{H}_{u}^{0}, \widetilde{H}_{d}^{0}\right)
$$

Using the LEP measurement of the invisible $Z$-decay width, the following constraint can be derived:

$$
\Gamma\left(Z \rightarrow \widetilde{\chi}_{i}^{0} \widetilde{\chi}_{j}^{0}\right)<3 \mathrm{MeV} \quad \text { if }\left(m_{\chi_{i}}+m_{\chi_{j}}\right)<m_{Z}
$$

Note that this constraint should apply to all light neutralinos satisfying this condition, not just the $\operatorname{LSP}\left(i=j=1\right.$ in eq. (3.4)). However, it is unlikely that decays such as $Z \rightarrow \widetilde{\chi}_{2}^{0} \widetilde{\chi}_{1}^{0}$ will be kinematically allowed, and in such cases, $\widetilde{\chi}_{2}^{0}$ will mostly decay to visible final states. Similarly, the decays $Z \rightarrow \widetilde{\nu}_{i} \widetilde{\nu}_{j}$ are not kinematically allowed for the parameter space examined here, and hence, they do not contribute to the purely invisible width of the $Z$-boson. 


\section{Exclusion limits from collider searches}

The experimental lower limits on the sparticle masses are usually quoted assuming gaugino and/or sfermion mass universality at the GUT scale. In a generic MSSM setup, most of these constraints can be relaxed, or even circumvented, for example in case of small mass splitting with the LSP or in case of small couplings to the SM vector bosons. Since we are interested in light neutralino LSPs here, we must carefully interpret the direct search limits in order to be able to include all the allowed pMSSM parameter space.

Neutralino. As already mentioned in section 1, there are no rigorous lower limits on the neutralino masses in the MSSM from direct collider searches. The LEP limits $[13,14,82]$

$$
m_{\widetilde{\chi}_{1}^{0}}>46 \mathrm{GeV}, m_{\widetilde{\chi}_{2}^{0}}>62.4 \mathrm{GeV}, m_{\widetilde{\chi}_{3}^{0}}>99.9 \mathrm{GeV}, m_{\widetilde{\chi}_{4}^{0}}>116.0 \mathrm{GeV}
$$

were derived assuming gaugino mass unification at the GUT scale, and hence, relating the neutralino mass to the chargino mass. Moreover, for a (mostly) bino-like neutralino, which is required to be the case for $m_{\widetilde{\chi}_{1}^{0}}<m_{Z} / 2$ in order to avoid the $Z$-width constraint, its production via $s$-channel exchange of $Z / \gamma^{*}$ is (negligible) absent. The $t$-channel production cross-section via selectron exchange is also expected to be small for selectron masses above the LEP limit (see below). Thus, we can easily satisfy the LEP upper limits on the neutralino pair-production cross sections $\sigma\left(e^{+} e^{-} \rightarrow \widetilde{\chi}_{i}^{0} \widetilde{\chi}_{j}^{0}\right)[82,83]$ for a mostly bino-like neutralino LSP. Similarly, the Tevatron [84] and LHC [85] SUSY searches for final states involving $Z$-bosons cannot constrain a bino-like neutralino.

Chargino. Charginos can be pair-produced at LEP via $s$-channel exchange of $Z / \gamma^{*}$ or $t$ channel exchange of electron-sneutrino, with destructive interference. It dominantly decays to $\ell \widetilde{\nu}$, if kinematically allowed. If not, the three-body decay to $f \bar{f}^{\prime} \widetilde{\chi}_{i}^{0}$ via virtual $W$-boson or sfermions becomes important in which case, the final state fermions $\left(f, \bar{f}^{\prime}\right)$ are dominantly leptonic (hadronic) if the sleptons are light (heavy). From the combined searches in fullyhadronic, semi-leptonic and fully-leptonic decay modes, LEP has derived a general lower limit of $103.5 \mathrm{GeV}[13,14]$ which is valid for pMSSM as well, except in corners of phase space where (i) the detection efficiencies are reduced, e.g., when the mass differences $\Delta m_{+}=$ $m_{\widetilde{\chi}_{1}^{ \pm}}-m_{\widetilde{\chi}_{1}^{0}}$ or $\Delta m_{\nu}=m_{\widetilde{\chi}_{1}^{ \pm}}-m_{\widetilde{\nu}}$ are very small (below a few $\mathrm{GeV}$ ); or (ii) the chargino production cross section is suppressed, e.g., when the electron sneutrino mass is small, thus leading to a destructive interference between $s$ - and $t$-channel Feynman diagrams. Dedicated searches for such scenarios have also been performed. For instance, for small $\Delta m_{+}<3 \mathrm{GeV}$ but with large sneutrino mass, the limit becomes $m_{\widetilde{\chi}_{1}^{ \pm}}>91.9 \mathrm{GeV}$ for degeneracy in the gaugino region $\left(\left|M_{1}\right| \sim\left|M_{2}\right| \ll|\mu|\right)$ while $m_{\widetilde{\chi}_{1}^{ \pm}}>92.4 \mathrm{GeV}$ for degeneracy in the higgsino region $\left(|\mu| \ll\left|M_{1}\right|,\left|M_{2}\right|\right)[13,14]$. Without assuming gaugino mass unification, a lower limit of $m_{\widetilde{\chi}_{1}^{ \pm}}>70 \mathrm{GeV}$ was set for any $\Delta m_{+}$and $m_{\widetilde{\nu}}>300 \mathrm{GeV}$. For smaller sneutrino masses, the sensitivity decreases due to the reduced pair production cross section and also due to reduced selection efficiency. In such situations where none of the above mass limits can apply, the generic lower limit of approximately $45 \mathrm{GeV}$, derived from the analysis of the $Z$-width, is still valid since this is independent of the field composition and of the decay modes of the charginos. Note that unlike neutralinos which couple to the $Z$-boson only via 
their higgsino component, the charginos couple to $Z$ via both their gaugino as well as higgsino components; so it is not possible to avoid the $Z$-width constraint for a light chargino.

Sneutrino. Light sneutrinos can only decay invisibly to $\nu \widetilde{\chi}_{1}^{0}$ unless the decays to charginos and heavier neutralinos are not kinematically suppressed. The invisible width of the $Z$-boson puts a lower limit on the left-sneutrino mass of $43.7 \mathrm{GeV}$, which improves slightly to $44.7 \mathrm{GeV}$ if all three sneutrinos are mass-degenerate. Note that the lightest left-sneutrino by itself cannot be a cold DM candidate [86, 87], and we must introduce a mixing with a SM singlet sneutrino to make it a viable DM candidate (see, e.g., [8894]). Since we are dealing here only with the MSSM field content and do not have a right-sneutrino component, we discard those points for which the sneutrino is the LSP.

Slepton. Studies of the $Z$-boson width and decays put a lower bound on the slepton masses $m_{\tilde{\ell}_{R(L)}}>40(41) \mathrm{GeV}$, independently of the decay modes for individual sleptons $(\widetilde{\ell}=$ $\widetilde{e}, \widetilde{\mu}, \widetilde{\tau})$. This limit improves to $43 \mathrm{GeV}$ if all the three slepton flavors are mass-degenerate. Tighter limits can be obtained assuming that sleptons are pair-produced at LEP, and each slepton dominantly decays to $\ell \widetilde{\chi}_{1}^{0}$, thus leading to two back-to-back leptons and missing transverse momentum. These limits are valid for a mass splitting $\Delta m_{\ell}=m_{\tilde{\ell}}-m_{\widetilde{\chi}_{1}^{0}}>$ $15 \mathrm{GeV}$ so that the final state leptons are not too soft. Moreover, the LEP results are interpreted assuming that only $\widetilde{\ell}_{R} \widetilde{\ell}_{R}$ production contributes, and hence, the limits are usually quoted for $\widetilde{\ell}_{R}$, since it is typically lighter than $\tilde{\ell}_{L}$ in most SUSY models, and has a weaker coupling to the $Z$-boson so that the limits are more conservative. This is a good approximation for selectrons and smuons, but not for staus which can have significant mixing between the flavor eigenstates $\widetilde{\tau}_{L}$ and $\widetilde{\tau}_{R}$. The most conservative limit on the mass of the lightest stau is obtained with a mixing angle $\theta_{\widetilde{\tau}} \simeq 52^{\circ}$ which minimizes the production cross section.

The slepton mass limits of $\mathcal{O}(100) \mathrm{GeV}$ quoted in ref. [13, 14] were derived under the assumption of gaugino mass unification at the GUT scale which was used to fix the masses and composition of neutralinos. It was also assumed that the slepton branching ratio to $\ell \widetilde{\chi}_{1}^{0}$ is nearly $100 \%$ which is a good approximation if the second lightest neutralino is heavy enough to suppress the cascade decay into $\ell \widetilde{\chi}_{2}^{0}$ followed by $\widetilde{\chi}_{2}^{0} \rightarrow f \bar{f} \widetilde{\chi}_{1}^{0}$ or $\widetilde{\chi}_{1}^{0} \gamma$. For smuons and staus, the LEP limits are independent of the MSSM parameters [95], and hence, directly applicable to our case. However, the selectron mass limit will be different if we do not assume gaugino mass unification due to a different production cross section involving the $t$-channel neutralino exchange, in addition to the usual $s$-channel $\gamma^{*} / Z$ exchange. Since there is no dedicated analysis of the LEP data addressing this issue available in the literature and this is beyond the scope of our present work, we only use the generic lower bound for $\widetilde{e}$ derived from the $Z$-width in our numerical analysis, but will also comment on the implications of the tighter selectron mass bound from LEP on our light neutralino scenario. We will also include the latest 95\% C.L. LHC exclusion limits for slepton pair production interpreted in the slepton-neutralino mass plane of pMSSM [96] which are applicable to both left- and right-handed selectrons and smuons.

Gluino and squarks. The current LEP limits on the squark masses are similar to the slepton mass limits of $\mathcal{O}(100) \mathrm{GeV}$. However, since squarks are colored objects, their 
production cross sections are much higher at hadron colliders. The highest sensitivity on squark and gluino production now comes from the LHC experiments. The generic lower limit on the first/second generation squark masses is $600-750 \mathrm{GeV}$ and on the gluino mass is $700-900 \mathrm{GeV}$, as set for simplified SUSY models by the ATLAS analysis of the $8 \mathrm{TeV}$ LHC data [26]. The corresponding CMS limits are very similar [27]. However, from the latest global fit of pMSSM after the LHC results [60] (see also ref. [97, 98] for an earlier global fit of pMSSM), the corresponding lower limit on squark mass is $m_{\widetilde{q}} \gtrsim 500 \mathrm{GeV}$, and hence, we use this value to constrain our pMSSM parameter space.

The LHC and Tevatron limits on the third generation squarks are usually weaker since the amount of bottom and top quark content in the proton is negligible, and hence, the direct production of bottom and top squark is suppressed with respect to the first/second generation squarks. The current exclusion limit for top squarks is between $300-600 \mathrm{GeV}$ from the LHC data, depending on the decay channel [26, 27]. Similar limits have been derived for the bottom squark as well. Following the latest global fit of pMSSM [60], we set the lower limits for light top squark and sbottom masses at $400 \mathrm{GeV}$ and $300 \mathrm{GeV}$, respectively. Note that these limits are applicable as long as the light stop/sbottom is not highly mass degenerate with the lightest neutralino (with $\Delta m<10 \mathrm{GeV}$ or so) which always turns out to be the case for our light neutralino solutions satisfying all the other constraints. Thus we do not have any light sbottom solutions as considered in ref. [39].

The lower limits on the sparticle masses derived from the above discussion are summarized in table 2. We emphasize here that for a light neutralino DM with $m_{\tilde{\chi}_{1}^{0}}<m_{Z} / 2$ as considered in our case, the dominant annihilation channels will be the $t$-channel processes mediated by light sfermions unless the $s$-channel $Z$-resonance or co-annihilation are effective. Hence, the lower limits on the chargino and sfermion masses as given in table 2 are crucial ingredients in our numerical analysis.

\section{$B_{s} \rightarrow \mu^{+} \mu^{-}$}

The rare decay $B_{s} \rightarrow \mu^{+} \mu^{-}$is known to be a sensitive channel for new physics since its $\mathrm{SM}$ predicted rate is small due to helicity suppression. Recently, the first evidence for this decay was observed by the LHCb collaboration with the measured branching ratio: $\mathcal{B}\left(B_{s} \rightarrow\right.$ $\left.\mu^{+} \mu^{-}\right)=\left(3.2_{-1.2}^{+1.5}\right) \times 10^{-9}[24]$ which is in excellent agreement with the SM prediction of $(3.23 \pm 0.27) \times 10^{-9}[25]$, thus raising some concerns for the "health" of SUSY. However, it must be noted that the upper limit derived from the latest LHCb result is actually slightly weaker than the earlier upper limit of $<4.5 \times 10^{-9}$ [99]. The effect of the new results is mostly felt in the large $\tan \beta>50$ regions of the MSSM parameter space [100] which are also strongly constrained from direct SUSY searches and the MSSM Higgs searches.

\section{$b \rightarrow s \gamma$}

We have also included the constraint from the radiative $B$-meson decay branching ratio, $\mathcal{B}(b \rightarrow s \gamma)=(3.55 \pm 0.24 \pm 0.09) \times 10^{-4}[101]$ which is somewhat higher than the SM prediction of $(3.15 \pm 0.23) \times 10^{-4}$ [102]. Thus large SUSY corrections are preferred which mainly occur for light chargino and top squarks and for large $\tan \beta$ [103]. 


\begin{tabular}{|c|c|c|}
\hline Particle & Mass limit $(\mathrm{GeV})$ & Validity Condition \\
\hline$\widetilde{\chi}_{1}^{ \pm}$ & 103.5 & $m_{\widetilde{\chi}_{1}^{+}}-m_{\widetilde{\chi}_{1}^{0}}>3 \mathrm{GeV}, m_{\widetilde{f}^{\prime}}>m_{\widetilde{\chi}^{ \pm}}$ \\
& 70 & $m_{\widetilde{\nu}}>300 \mathrm{GeV},|\mu| \geq\left|M_{2}\right|$ \\
& 45 & generic LEP bound \\
\hline$\widetilde{\mu}_{R}$ & 88 & $m_{\widetilde{\mu}_{R}}-m_{\widetilde{\chi}_{1}^{0}}>15 \mathrm{GeV}, \mathrm{BR}\left(\widetilde{\mu} \rightarrow \mu \widetilde{\chi}_{1}^{0}\right)=1$ \\
$\widetilde{\tau}_{1}$ & 76 & $m_{\widetilde{\tau}_{1}}-m_{\widetilde{\chi}_{1}^{0}}>15 \mathrm{GeV}, \mathrm{BR}\left(\widetilde{\tau}_{1} \rightarrow \tau \widetilde{\chi}_{1}^{0}\right)=1$ \\
$\widetilde{e}_{R}$ & 95 & $m_{\widetilde{e}_{R}}-m_{\widetilde{\chi}_{1}^{0}}>15 \mathrm{GeV}, \mathrm{BR}\left(\widetilde{e} \rightarrow e \widetilde{\chi}_{1}^{0}\right)=1$, \\
& & $\mu=-200 \mathrm{GeV}, \tan \beta=2$ \\
\hline$\widetilde{\ell}_{R}(L)$ & $40(41)$ & generic LEP bound \\
$\widetilde{\nu}$ & 43.7 & generic LEP bound \\
\hline$\widetilde{g}$ & 800 & \\
$\widetilde{q}$ & 500 & \\
$\widetilde{t}_{1}$ & 400 & \\
$\widetilde{b}_{1}$ & 300 & \\
\hline
\end{tabular}

Table 2. The lower limits on the sparticle masses used in our numerical analysis. The chargino and slepton mass limits are derived from the LEP data $[13,14]$ while the squark and gluino mass limits are derived from the LHC data $[26,27]$ which now supersede the LEP as well as the Tevatron $[69,70]$ limits.

$(g-2)_{\mu}$

Another important constraint comes from the muon anomalous magnetic moment $a_{\mu} \equiv(g-$ $2)_{\mu} / 2$ which gives a more than $3 \sigma$ discrepancy with the SM prediction: $\delta a_{\mu}=(26.1 \pm 8.0) \times$ $10^{-10}$ [104]. The SUSY contribution to $\delta a_{\mu}$ can explain this discrepancy with relatively light smuons and/or large $\tan \beta$ [105-107], and $\mu<0$ region of the SUSY parameter space is strongly disfavored (unless the electroweak gaugino masses $M_{1}, M_{2}<0$ ).

\section{Higgs sector}

For the light $C P$-even Higgs mass, we have chosen the value of $m_{h}=(125 \pm 2) \mathrm{GeV}$, following the latest best-fit mass measurements of the Higgs-like particle discovered at the LHC: $(125.2 \pm 0.3 \pm 0.6) \mathrm{GeV}$ (ATLAS) [108] and (125.8 $\pm 0.4 \pm 0.4) \mathrm{GeV}$ (CMS) [109]. For the other MSSM Higgs bosons, we ensure that all our allowed points satisfy the latest LHC constraints on the $m_{A}-\tan \beta$ plane $[110,111]$ and on the MSSM charged Higgs mass $[112,113]$ which are related to each other at tree-level by $m_{H^{ \pm}}^{2}=m_{A}^{2}+m_{W}^{2}$. Note that the non-decoupling region with light $m_{A} \sim 95-130 \mathrm{GeV}$, almost mass-degenerate with the light $C P$-even Higgs, and with the heavy $C P$-even Higgs SM-like, is disfavored $[114,115]$ by the latest LHC Higgs data and flavor constraints, especially the $B_{s} \rightarrow \mu^{+} \mu^{-}$and $b \rightarrow s \gamma$. Hence, we work in the decoupling region with $m_{A}>300 \mathrm{GeV}$ with the light $C P$-even Higgs SM-like with mass in the vicinity of $125 \mathrm{GeV}$, and with the heavy $C P$-even Higgs nearly mass-degenerate with the $C P$-odd Higgs. Also note that for 
a mostly bino-like neutralino, the $h \widetilde{\chi}_{1}^{0} \bar{\chi}_{1}^{0}$ coupling is small enough to satisfy the current global limit on the Higgs invisible decay branching ratio: $\mathrm{BR}_{\mathrm{inv}}<0.28$ at $95 \% \mathrm{CL}$ [116].

\section{Dark matter constraints}

The latest results from Planck give the current relic density of the cold dark matter content in our universe to be $\Omega_{\chi} h^{2}=0.1199 \pm 0.0027$ at $68 \%$ CL [72]. The corresponding value from the 9-year WMAP data is $\Omega_{\chi} h^{2}=0.1148 \pm 0.0019$ [71]. For the relic density of the neutralino DM in our case, we only require it to satisfy the WMAP $2 \sigma$ upper bound combined with $10 \%$ theoretical uncertainty: $\Omega_{\widetilde{\chi}_{1}} h^{2}<0.138$ which also encompasses the latest observed value from Planck. The cases where the neutralino relic density is below the corresponding WMAP lower bound, $\Omega_{\widetilde{\chi}_{1}^{0}} h^{2}<0.091$, could account for the correct relic density by alternative mechanisms of regeneration (see e.g., [117-119]), or by invoking a multi-component DM scenario (see e.g., [120-129]).

As far as the DM direct detection constraints are concerned, since there is no unanimous upper bound on the direct detection cross section, we do not put this constraint a priori on the model parameter space. However, as we will see later, most of the allowed parameter space satisfying the other constraints also satisfy the most stringent upper limit on the spin-independent DM-nucleon scattering cross section set by the XENON100 experiment [10]. Note that for $m_{\chi}>10 \mathrm{GeV}$, the spin-independent direct detection constraints are more stringent than the collider constraints from mono-jet $[130,131]$ and isolated monophoton $[132,133]$ searches at the LHC as well as from the LEP mono-photon data [134].

Complementary to the direct detection constraints, there exist indirect detection constraints which are mostly sensitive to light WIMPs annihilating to SM fermions which eventually lead to gamma-ray signals. A lower limit of $m_{\widetilde{\chi}_{1}^{0}} \gtrsim 10 \mathrm{GeV}$ was derived from the CMB constraints $[135-140]^{3}$ for DM candidates with a velocity-independent annihilation cross section of $\left\langle\sigma_{a} v\right\rangle=3 \times 10^{-26} \mathrm{~cm}^{3} \cdot \mathrm{s}^{-1}$. Under the same assumption, the Fermi-LAT data put lower bounds of $m_{\widetilde{\chi}_{1}^{0}} \gtrsim 27 \mathrm{GeV}$ for annihilation to $b \bar{b}$ channel and $m_{\widetilde{\chi}_{1}^{0}} \gtrsim 37 \mathrm{GeV}$ for $\tau^{+} \tau^{-}$channel [142]. However, these bounds can be relaxed if we include the velocitydependent contributions, as shown for pMSSM in ref. [143]. In our numerical analysis, we include the latest Fermi-LAT 95\% CL upper limit on the integrated $\gamma$-ray flux from spectral line searches in the Milky Way galaxy: $\phi_{\gamma}<4 \times 10^{-10} \mathrm{~cm}^{-2} \cdot \mathrm{s}^{-1}$ [73] for high latitude $\left(|b|>10^{\circ}\right)$ plus a $20^{\circ} \times 20^{\circ}$ square at the galactic center and for $7-200 \mathrm{GeV}$ energy range.

The various experimental constraints discussed above and used in our numerical analysis, in addition to the sparticle mass limits listed in table 2, are summarized in table 3.

\section{Results}

In order to scan the 19-dimensional pMSSM parameter space more efficiently while satisfying all the constraints listed in tables 2 and 3, we have performed a MCMC analysis using a Gaussian distribution of likelihood function: $f\left(x, x_{0}, \sigma\right)=\exp \left[-\left(x-x_{0}\right)^{2} / 2 \sigma^{2}\right]$ for

\footnotetext{
${ }^{3}$ Similar limits have also been derived from BBN constraints [141].
} 


\begin{tabular}{|c|c|}
\hline Parameter & Constraint \\
\hline$m_{h}$ & $(125 \pm 2) \mathrm{GeV}$ \\
$\Gamma_{Z}^{\text {invisible }}$ & $<3 \mathrm{MeV}$ \\
$\Omega_{\widetilde{\chi}_{1}^{0}} h^{2}$ & $<0.138$ \\
$\phi_{\gamma}$ & $<4 \times 10^{-10} \mathrm{~cm}^{-2} \cdot \mathrm{s}^{-1}$ \\
$\mathcal{B}\left(B_{s} \rightarrow \mu^{+} \mu^{-}\right)$ & $\left(3.2_{-1.2}^{+1.5}\right) \times 10^{-9}$ \\
$\mathcal{B}(b \rightarrow s \gamma)$ & $(3.55 \pm 0.26) \times 10^{-4}$ \\
$\delta a_{\mu}$ & $(26.1 \pm 8.0) \times 10^{-10}$ \\
\hline
\end{tabular}

Table 3. The relevant experimental constraints used in our analysis, in addition to those listed in table 2.

all the observables, with a preferred value $x_{0} \pm \sigma$. We have used CaclHEP2.3 [144] and micrOMEGAs2 . 4 [145-147] to compute all the observables, together with SoftSUSY [148] for calculating the particle spectrum.

First we discuss our MCMC scan results for the relic density of a light neutralino DM candidate as shown in figure 1, which was obtained by numerically solving the Boltzmann equation using micrOMEGAS [145]. We require all the allowed points (shown as circles) to satisfy the experimental constraints given in table 3, along with the LEP limits on sparticle masses given in table 2. The latest LHC results put much tighter bounds on the strongly interacting squarks and gluinos and further eliminate some of these otherwise allowed parameter space, as shown by the starred points in figure 1. The WMAP-9 $2 \sigma$ band is shown in grey, whereas the latest Planck result is shown as dark shaded region. We find that light neutralinos with mass as low as $10 \mathrm{GeV}$ are still allowed, though severely fine-tuned with the electroweak fine-tuning measure defined by eq. (2.4): $\Delta_{\text {tot }} \gg 1$. This can be understood as follows by analyzing the gaugino and higgsino components of the lightest neutralino as well as its dominant annihilation channels.

The bino, wino and higgsino fractions of the lightest neutralino for all the allowed points in our pMSSM parameter space are shown in figure 2. We reproduce the well-known result that the lightest neutralino is mostly bino-like for masses below $m_{Z} / 2$, mainly due to the invisible $Z$-decay width constraint in eq. (3.4). However, a purely bino DM tends to overclose the universe unless it has an efficient annihilation channel to reach up to the thermal WIMP annihilation rate of $3 \times 10^{-26} \mathrm{~cm}^{3} \cdot \mathrm{s}^{-1}$. One possibility is to consider a "well-tempered" neutralino [149] which corresponds to the boundary between a pure bino and a pure higgsino or wino. Another possibility to reduce the bino relic density is by annihilation via the $t$-channel slepton exchange (the so-called "bulk region") which is efficient for light sleptons, or by using co-annihilation with a light slepton, squark, chargino or second-lightest neutralino in configurations where such light sparticles are not yet excluded by experimental searches. We find that most of the points with $m_{\widetilde{\chi}_{1}^{0}}$ close to $45 \mathrm{GeV}$ can have either slepton co-annihilation or a resonant $Z$-annihilation due to a non-negligible higgsino component, and hence, can easily satisfy the WMAP upper limit 




Figure 1. The relic density of a light neutralino DM in pMSSM satisfying all the experimental constraints discussed in section 3. The color-coding denotes the fine-tuning measure defined by eq. (2.4). The points denoted by circles satisfy all the experimental constraints, except that the squark masses are only required to satisfy the LEP lower limits. For the starred points (a subset of the circled points), the corresponding squark masses satisfy the latest LHC constraints. The top (bottom) grey horizontal line shows the $2 \sigma$ upper (lower) limit of the cold dark matter relic density from WMAP-9 data, whereas the black (shaded) region shows the $1 \sigma$ allowed range from the recent Planck data.



Figure 2. The gaugino $\left(\widetilde{B}, \widetilde{W}^{0}\right)$ and higgsino $\left(\widetilde{H}_{d}^{0}, \widetilde{H}_{u}^{0}\right)$ components of the lightest neutralino in our pMSSM parameter scan.

on the relic density. These points are also less fine-tuned. On the other hand, the light neutralino DM points in the $10-30 \mathrm{GeV}$ range as shown in figure 1 have to be mostly bino-like and lie in the bulk region, thus leading to significant fine-tuning. Note that in the latter case, the next-to-lightest supersymmetric particle (NLSP) masses are much higher than the LSP mass, thus eliminating the possibility of a co-annihilation.

This is further clarified in figure 3 where we show the various NLSPs and their masses as a function of the lightest neutralino mass. We see that all the allowed points 




Figure 3. The various NLSP masses as a function of the LSP mass for the allowed points (circles) shown in figure 1 . The LEP exclusion regions strictly applicable for $\widetilde{\mu}_{R}$ (red shaded) and $\widetilde{\tau}_{1}$ (green shaded) and the LHC exclusion region for $\tilde{\ell}(l=e, \mu)$ (blue shaded) are also shown.

with $m_{\widetilde{\chi}_{1}^{0}}<30 \mathrm{GeV}$ have a charged slepton NLSP with mass below $100 \mathrm{GeV}$. Especially the points with a light stau are severely fine-tuned since they usually require a mass suppression by the off-diagonal elements in the slepton mass matrix, or a large $\mu$-term. We also show in figure 3 the LEP exclusion regions in the charged slepton-neutralino mass plane, derived under the assumption of gaugino mass unification $[13,14]$. The limits for light smuons and staus are still applicable to the pMSSM case as long as $\Delta m_{\ell}>15 \mathrm{GeV}$, but not directly to light selectrons if we assume non-universal gaugino masses, and hence, can still allow the low neutralino mass regime. The latest 95\% C.L. ATLAS exclusion limits [96] are also shown in figure 3 which were derived from searches for direct slepton (selectron and smuon) pair production and interpreted in the pMSSM. A similar dedicated analysis of the LEP data is required in order to completely rule out the light selectrons, and hence, the lightest neutralino DM mass below $30 \mathrm{GeV}$ for the pMSSM scenario.

The neutralino DM-nucleon spin-independent scattering cross-sections for the allowed points is shown in figure 4 . The points corresponding to the observed relic density within the WMAP band in figure 1 are encircled. As mentioned earlier, the remaining points can also account for the correct relic density if we assume $100 \%$ regeneration at late times (see e.g., [117-119]). Another possibility is to invoke a multiple DM scenario (see e.g., [120129]) in which case the neutralino DM considered here will only constitute a fraction of the total observed DM density, and we must scale the neutralino DM density appropriately in order to calculate the neutralino-nucleon scattering cross section. Since the cross section depends linearly on the DM density, we use a rescaling factor of $r_{\chi} \equiv \Omega_{\widetilde{\chi}_{1}^{0}} / \Omega_{\text {observed }}$, where for concreteness, we take the Planck central value for $\Omega_{\text {observed }} h^{2}=0.12$. For the DM density distribution in the galactic halo, we have used the NFW profile [150, 151], as implemented in micrOMEGAs_2.4 [146]. The results with and without rescaling of the DM density are shown for comparison in figure 4. A comparison of the two panels in figure 4 shows that the light neutralino solutions (with large fine-tuning) are mostly unaffected by 

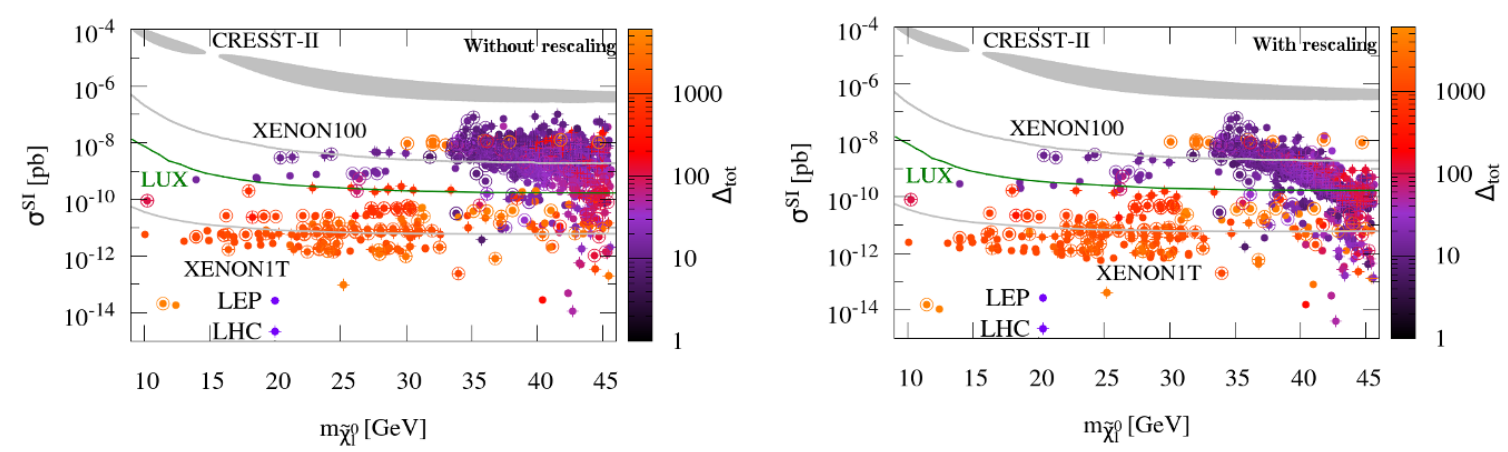

Figure 4. The spin-independent direct detection cross-section values for the allowed points in our pMSSM scan. The color-coding and labeling of the points are same as in figure 1. The circled points correspond to those within the WMAP allowed band in figure 1. The left (right) panel shows the cross section without (with) rescaling of the DM density. The current upper limit from the XENON100 experiment and the projected limits from LUX and XENON1T experiments are shown as solid lines. The $2 \sigma$-preferred range of CRESST-II is shown as the shaded region.

the rescaling since these points yield a relic density value more than $10 \%$ of the observed value (see figure 1). It is interesting to note that most of the allowed region in figure 1 with low fine-tuning lead to a higher scattering cross section via $Z$-boson exchange and are already ruled out, even after rescaling, by the latest XENON100 data [10] or will be ruled out by the projected limit of XENON1T experiment [74] (and also LUX [75]) if they still get a null result. The points which survive with rescaling must be part of some multi-component DM scenario. In the light of the recent claims for positive hints of a light DM from some experiments, it is worth mentioning here that a few of our solutions with $\sigma^{\mathrm{SI}} \sim 10^{-7} \mathrm{pb}$ are in the vicinity of the $2 \sigma$ preferred range of the CRESST-II results [9], but not compatible with the favored regions of DAMA [6, 7] or CoGeNT [8].

Figure 5 shows the integrated photon flux from spectral lines due to neutralino DM annihilation in the galactic halo. We have assumed the NFW profile for the DM density distribution, as implemented in micrOMEGAs_2.4 [147]. We do not consider galactic sub-structures or clumpy DM configurations since a proper analysis of these effects would require a detailed numerical simulation well beyond the scope of this work. As in figure 4 for scattering cross-section, we have shown the fluxes for both the cases - without and with scaling of the neutralino DM density. In the latter case, we have used the scaling factor $r_{\chi}^{2}$ for the thermally-averaged annihilation cross section $\left\langle\sigma_{a} v\right\rangle$ when the neutralino DM relic density is below the observed value. The light neutralino solutions with a relic density more than $10 \%$ of the observed value (cf. figure 1) are mostly unaffected by the rescaling. Note that since the LSP in our case is mostly bino-like with heavy squarks and higgsinos and the charginos are not mass-degenerate with the LSP, the annihilation to photons is loop-suppressed, and hence, the photon line emission will be small. It is clear from figure 5 that the current sensitivity of Fermi-LAT [142] still leaves all of our allowed parameter space untouched. The future data from ongoing Fermi-LAT and next generation gamma-ray searches might be able to probe our allowed parameter space with a photon line signal [152]. 

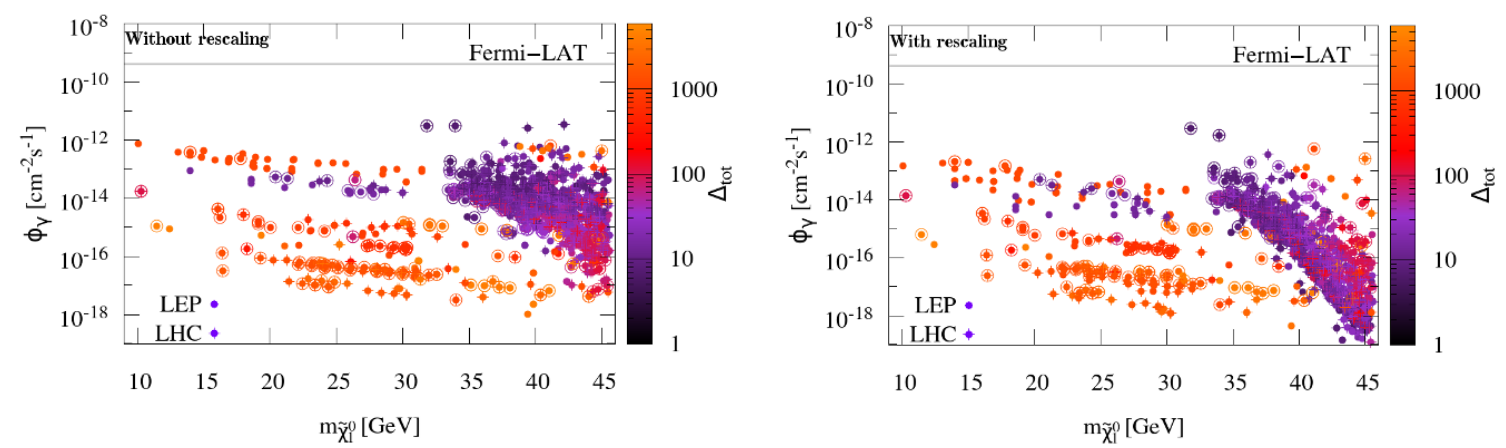

Figure 5. The integrated photon flux from annihilation of the neutralino DM as a function of its mass. The color-coding and labeling of the points are same as in figure 4 . The solid horizontal line shows the current upper limit from the Fermi-LAT data.


Figure 6. The allowed parameter space for some of the relevant pMSSM parameters consistent with a light neutralino DM. The color-coding and labeling of the points are the same as in figure 1.

For completeness, we also show in figure 6 some other relevant pMSSM parameters with respect to the lightest neutralino mass. The $m_{A}-\tan \beta$ parameter space is mostly consistent with the latest MSSM Higgs sector limits from the LHC [110, 111]. As for the bino mass parameter $M_{1}$, it is clear that a relatively small value of $\left|M_{1}\right|<100 \mathrm{GeV}$ is preferred to obtain a light bino-like neutralino LSP. Finally, as is well-known, a relatively large value of $\left|A_{t}\right|$ is required in order to enhance the radiative corrections for the light $C P$-even Higgs mass to be consistent with the LHC-preferred value of $125 \pm 2 \mathrm{GeV}$.

Finally, we wish to point out that the allowed sparticle spectra discussed here with mostly heavy squarks, and with light sleptons, chargino and bino-like neutralino LSP, are 
also crucial for explaining the muon $(g-2)$ anomaly [153], while simultaneously satisfying all the other experimental constraints. Light staus with sizable left-right mixing can also lead to an enhanced $h \rightarrow \gamma \gamma$ decay rate [154-156], which might be able to explain the persistent excess in the Higgs signal strength in this channel: $1.65 \pm 0.24$ (stat) ${ }_{-0.18}^{+0.25}$ (syst) [157].

\section{Conclusion}

We have studied the naturalness of a light neutralino dark matter candidate in the MSSM in the light of the latest results from the collider (LEP, Tevatron and LHC), flavor (LHCb) and dark matter (XENON100, WMAP-9, Planck) sectors. In particular, keeping in mind the recent positive hints for a light DM below $45 \mathrm{GeV}$ in some direct detection experiments and the null results from SUSY searches at the LHC, we perform a dedicated study focusing on the possibility of a light DM candidate in the form of the lightest neutralino in the pMSSM, also analyzing the naturalness of such a scenario. We include all the new results from the ongoing LHC experiments in our analysis, such as the null results from the SUSY searches, the observation of a Higgs-like particle, the observation of the rare meson decay $B_{s} \rightarrow \mu^{+} \mu^{-}$, and the updated constraints on the MSSM Higgs sector. We also take into account the 9-year WMAP results as well as the recently released Planck results for the DM relic density and the Fermi-LAT data for the integrated photon flux. In order to efficiently scan over the 19-dimensional pMSSM parameter space, we perform a MCMC likelihood analysis focusing on a light neutralino with mass below the LEP bound of $46 \mathrm{GeV}$ (applicable to gaugino mass unification models).

We find that a light neutralino DM with mass as low as $10 \mathrm{GeV}$ is still allowed in the pMSSM, while satisfying all the existing experimental constraints. However, such neutralinos which are required to be mostly bino-like are severely fine-tuned and require the existence of light sleptons with mass below $100 \mathrm{GeV}$ in order to provide an efficient annihilation channel to reduce the DM relic density below the observed upper limit. Such light smuons and staus are excluded from LEP searches while light selectrons are excluded only if we assume gaugino mass unification. A dedicated analysis of the LEP data in the context of a pMSSM scenario could completely eliminate the possibility of a light neutralino DM in the mass range of $10-30 \mathrm{GeV}$. We also find that for the allowed parameter space, other possible solutions in this mass range as discussed in the literature (e.g., light sbottom NLSP) are now excluded mainly due to the latest LHC results on the strongly interacting sfermion sector.

On the other hand, low fine-tuning regions can be obtained around $m_{\widetilde{\chi}_{1}^{0}}=45 \mathrm{GeV}$ where the resonant annihilation via the $s$-channel $Z$-exchange is possible for a bino-higgsino mixture of neutralino LSP. However, such regions also predict a higher spin-independent DM-nucleon scattering cross section, and are mostly excluded by the recent XENON100 limits. The remaining such points are within the reach of the XENON1T projected limits.

In conclusion, while a light neutralino $\mathrm{DM}$ with mass below $46 \mathrm{GeV}$ has been conclusively ruled out in MSSM with gaugino mass unification by LEP searches, such a possibility in a general version of MSSM is of enormous interest in the light of the recent claims for a positive signal in some DM direct detection experiments. Taking into account the latest experimental results from collider, flavor, dark matter and 
astrophysical/cosmological sectors, we show that such a light neutralino DM scenario is also getting highly constrained in MSSM without gaugino mass unification. However, within a pMSSM scenario, there still exists some parameter space for light neutralino DM which could be completely probed by a dedicated analysis of the existing experimental data, in combination with the ongoing searches.

Note added. After the submission of our paper, another DM direct detection experiment, namely, CDMS-II [158], has reported three WIMP-candidate events with an expected background of 0.7 events. Their best-fit WIMP mass is $8.6 \mathrm{GeV}$ with the WIMP-nucleon cross section of $1.9 \times 10^{-5} \mathrm{pb}$. This bolsters our motivation in this work to examine the allowed parameter space for a light neutralino DM candidate in the MSSM.

\section{Acknowledgments}

We thank Matthew Dolan and Patrick Janot for valuable discussions and comments on the draft, and Jonathan Da Silva for useful discussions regarding the MCMC analysis. CB is supported by the ERC advanced grant 'DARK' at IAP, Paris. The work of PSBD and AM is supported by the Lancaster-Manchester-Sheffield Consortium for Fundamental Physics under STFC grant ST/J000418/1. EP is supported by STFC ST/J501074.

Open Access. This article is distributed under the terms of the Creative Commons Attribution License which permits any use, distribution and reproduction in any medium, provided the original author(s) and source are credited.

\section{References}

[1] M. Drees, R.M. Godbole and P. Roy, Theory and phenomenology of sparticles, World Scientific, Singapore (2004).

[2] H. Baer and X. Tata, Weak scale supersymmetry, Cambridge University Press, Cambridge U.K. (2006).

[3] G. Jungman, M. Kamionkowski and K. Griest, Supersymmetric dark matter, Phys. Rept. 267 (1996) 195 [hep-ph/9506380] [INSPIRE].

[4] K. Griest and M. Kamionkowski, Supersymmetric dark matter, Phys. Rept. 333 (2000) 167 [INSPIRE].

[5] Particle Data Group collaboration, J. Beringer et al., Review of particle physics (RPP), Phys. Rev. D 86 (2012) 010001 [inSPIRE].

[6] DAMA collaboration, R. Bernabei et al., First results from DAMA/LIBRA and the combined results with DAMA/NaI, Eur. Phys. J. C 56 (2008) 333 [arXiv:0804.2741] [INSPIRE].

[7] DAMA and LIBRA collaborations, R. Bernabei et al., New results from DAMA/LIBRA, Eur. Phys. J. C 67 (2010) 39 [arXiv:1002.1028] [InSPIRE].

[8] CoGeNT collaboration, C. Aalseth et al., Results from a search for light-mass dark matter with a P-type point contact germanium detector, Phys. Rev. Lett. 106 (2011) 131301 [arXiv: 1002.4703] [INSPIRE]. 
[9] G. Angloher et al., Results from $730 \mathrm{~kg}$ days of the CRESST-II dark matter search, Eur. Phys. J. C 72 (2012) 1971 [arXiv:1109.0702] [INSPIRE].

[10] XENON100 collaboration, E. Aprile et al., Dark matter results from 225 live days of XENON100 data, Phys. Rev. Lett. 109 (2012) 181301 [arXiv:1207.5988] [INSPIRE].

[11] TEXONO collaboration, H. Li et al., Limits on spin-independent couplings of WIMP dark matter with a p-type point-contact germanium detector, arXiv:1303.0925 [INSPIRE].

[12] S.P. Martin, A supersymmetry primer, in Perspectives on supersymmetry II, G.L. Kane ed., pg. 1 [hep-ph/9709356] [INSPIRE].

[13] DELPHI collaboration, J. Abdallah et al., Searches for supersymmetric particles in $e^{+} e^{-}$ collisions up to $208 \mathrm{GeV}$ and interpretation of the results within the MSSM, Eur. Phys. J. C 31 (2003) 421 [hep-ex/0311019] [INSPIRE].

[14] Joint LEP 2, SUSY Working Group, ALEPH, DELPHI, L3 and OPAL experiments webpage, http://lepsusy.web.cern.ch/lepsusy/.

[15] P. Nath, R. Arnowitt and A.H. Chamseddine, Applied $N=1$ supergravity, World Scientific, Singapore (1984).

[16] H.K. Dreiner et al., Mass bounds on a very light neutralino, Eur. Phys. J. C 62 (2009) 547 [arXiv:0901.3485] [INSPIRE].

[17] D. Hooper and T. Plehn, Supersymmetric dark matter: how light can the LSP be?, Phys. Lett. B 562 (2003) 18 [hep-ph/0212226] [INSPIRE].

[18] A. Bottino, F. Donato, N. Fornengo and S. Scopel, Lower bound on the neutralino mass from new data on CMB and implications for relic neutralinos, Phys. Rev. D 68 (2003) 043506 [hep-ph/0304080] [INSPIRE].

[19] G. Bélanger, F. Boudjema, A. Cottrant, A. Pukhov and S. Rosier-Lees, Lower limit on the neutralino mass in the general MSSM, JHEP 03 (2004) 012 [hep-ph/0310037] [INSPIRE].

[20] J.S. Lee and S. Scopel, Lightest Higgs boson and relic neutralino in the MSSM with CP-violation, Phys. Rev. D 75 (2007) 075001 [hep-ph/0701221] [INSPIRE].

[21] ATLAS collaboration, Observation of a new particle in the search for the Standard Model Higgs boson with the ATLAS detector at the LHC, Phys. Lett. B 716 (2012) 1 [arXiv: 1207.7214] [INSPIRE].

[22] CMS collaboration, Observation of a new boson at a mass of $125 \mathrm{GeV}$ with the CMS experiment at the LHC, Phys. Lett. B 716 (2012) 30 [arXiv:1207.7235] [INSPIRE].

[23] A. Djouadi, The anatomy of electro-weak symmetry breaking. II. The Higgs bosons in the minimal supersymmetric model, Phys. Rept. 459 (2008) 1 [hep-ph/0503173] [INSPIRE].

[24] LHCB collaboration, First evidence for the decay $B_{s}^{0} \rightarrow \mu^{+} \mu^{-}$, Phys. Rev. Lett. 110 (2013) 021801 [arXiv: 1211.2674] [INSPIRE].

[25] A.J. Buras, J. Girrbach, D. Guadagnoli and G. Isidori, On the Standard Model prediction for $B R\left(B_{s, d} \rightarrow \mu^{+} \mu^{-}\right)$, Eur. Phys. J. C 72 (2012) 2172 [arXiv:1208.0934] [INSPIRE].

[26] ATLAS Supersymmetry (SUSY) searches webpage, https://twiki.cern.ch/twiki/bin/view/AtlasPublic/SupersymmetryPublicResults.

[27] CMS Supersymmetry physics results webpage, https://twiki.cern.ch/twiki/bin/view/CMSPublic/PhysicsResultsSUS. 
[28] ATLAS collaboration, Search for neutral MSSM Higgs bosons decaying to $\tau^{+} \tau^{-}$pairs in proton-proton collisions at $\sqrt{s}=7 \mathrm{TeV}$ with the ATLAS detector, Phys. Lett. B 705 (2011) 174 [arXiv:1107.5003] [INSPIRE].

[29] ATLAS collaboration, Search for charged Higgs bosons decaying via $H^{+} \rightarrow \tau \nu$ in top quark pair events using pp collision data at $\sqrt{s}=7 \mathrm{TeV}$ with the ATLAS detector, JHEP 06 (2012) 039 [arXiv: 1204.2760] [inSPIRE].

[30] CMS collaboration, Search for neutral Higgs bosons decaying to $\tau$ pairs in pp collisions at $\sqrt{s}=7 \mathrm{TeV}$, CMS-PAS-HIG-11-020, CERN, Geneva Switzerland (2011).

[31] D. Feldman, Z. Liu and P. Nath, Low mass neutralino dark matter in the MSSM with constraints from $B_{s} \rightarrow \mu^{+} \mu^{-}$and Higgs search limits, Phys. Rev. D 81 (2010) 117701 [arXiv: 1003.0437] [INSPIRE].

[32] E. Kuflik, A. Pierce and K.M. Zurek, Light neutralinos with large scattering cross sections in the minimal supersymmetric Standard Model, Phys. Rev. D 81 (2010) 111701 [arXiv: 1003.0682] [INSPIRE].

[33] D.A. Vasquez, G. Bélanger, C. Boehm, A. Pukhov and J. Silk, Can neutralinos in the MSSM and NMSSM scenarios still be light?, Phys. Rev. D 82 (2010) 115027 [arXiv: 1009.4380] [inSPIRE].

[34] D. Albornoz Vasquez, G. Bélanger and C. Boehm, Revisiting light neutralino scenarios in the MSSM, Phys. Rev. D 84 (2011) 095015 [arXiv:1108.1338] [InSPIRE].

[35] N. Fornengo, S. Scopel and A. Bottino, Discussing direct search of dark matter particles in the minimal supersymmetric extension of the Standard Model with light neutralinos, Phys. Rev. D 83 (2011) 015001 [arXiv: 1011.4743] [INSPIRE].

[36] A. Bottino, N. Fornengo and S. Scopel, Phenomenology of light neutralinos in view of recent results at the CERN Large Hadron Collider, Phys. Rev. D 85 (2012) 095013 [arXiv:1112.5666] [INSPIRE].

[37] L. Calibbi, T. Ota and Y. Takanishi, Light neutralino in the MSSM: a playground for dark matter, flavor physics and collider experiments, JHEP 07 (2011) 013 [arXiv:1104.1134] [INSPIRE].

[38] A. Choudhury and A. Datta, Many faces of low mass neutralino dark matter in the unconstrained MSSM, LHC data and new signals, JHEP 06 (2012) 006 [arXiv:1203.4106] [INSPIRE].

[39] A. Arbey, M. Battaglia and F. Mahmoudi, Light neutralino dark matter in the pMSSM: implications of LEP, LHC and dark matter searches on SUSY particle spectra, Eur. Phys. J. C 72 (2012) 2169 [arXiv:1205.2557] [INSPIRE].

[40] C. Boehm, J. Da Silva, A. Mazumdar and E. Pukartas, Probing the supersymmetric inflaton and dark matter link via the CMB, LHC and XENON1T experiments, Phys. Rev. D 87 (2013) 023529 [arXiv: 1205.2815] [INSPIRE].

[41] G. Bélanger, S. Biswas, C. Boehm and B. Mukhopadhyaya, Light neutralino dark matter in the MSSM and its implication for LHC searches for staus, JHEP 12 (2012) 076 [arXiv:1206.5404] [INSPIRE].

[42] P. Grothaus, M. Lindner and Y. Takanishi, Naturalness of neutralino dark matter, arXiv:1207.4434 [INSPIRE]. 
[43] W. Altmannshofer, M. Carena, N.R. Shah and F. Yu, Indirect probes of the MSSM after the Higgs discovery, JHEP 01 (2013) 160 [arXiv:1211.1976] [INSPIRE].

[44] C. Cheung, L.J. Hall, D. Pinner and J.T. Ruderman, Prospects and blind spots for neutralino dark matter, JHEP 05 (2013) 100 [arXiv:1211.4873] [INSPIRE].

[45] T. Han, Z. Liu and A. Natarajan, Dark matter and Higgs bosons in the MSSM,

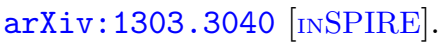

[46] J.R. Ellis, K. Enqvist, D.V. Nanopoulos and F. Zwirner, Observables in low-energy superstring models, Mod. Phys. Lett. A 1 (1986) 57 [INSPIRE].

[47] R. Barbieri and G. Giudice, Upper bounds on supersymmetric particle masses, Nucl. Phys. B 306 (1988) 63 [INSPIRE].

[48] J.R. Ellis, S. King and J. Roberts, The fine-tuning price of neutralino dark matter in models with non-universal Higgs masses, JHEP 04 (2008) 099 [arXiv:0711.2741] [INSPIRE].

[49] D. Horton and G. Ross, Naturalness and focus points with non-universal gaugino masses, Nucl. Phys. B 830 (2010) 221 [arXiv:0908.0857] [INSPIRE].

[50] M. Perelstein and B. Shakya, Fine-tuning implications of direct dark matter searches in the MSSM, JHEP 10 (2011) 142 [arXiv:1107.5048] [INSPIRE].

[51] S. Amsel, K. Freese and P. Sandick, Probing EWSB naturalness in unified SUSY models with dark matter, JHEP 11 (2011) 110 [arXiv:1108.0448] [INSPIRE].

[52] A. Arbey, M. Battaglia and F. Mahmoudi, Implications of LHC searches on SUSY particle spectra: the pMSSM parameter space with neutralino dark matter, Eur. Phys. J. C 72 (2012) 1847 [arXiv: 1110.3726] [INSPIRE].

[53] A. Arbey, M. Battaglia and F. Mahmoudi, Constraints on the MSSM from the Higgs sector: a pMSSM study of Higgs searches, $B_{s}^{0} \rightarrow \mu^{+} \mu^{-}$and dark matter direct detection, Eur. Phys. J. C 72 (2012) 1906 [arXiv:1112.3032] [InSPIRE].

[54] P. Bechtle et al., Constrained supersymmetry after two years of LHC data: a global view with fittino, JHEP 06 (2012) 098 [arXiv:1204.4199] [INSPIRE].

[55] S. Fichet, Quantified naturalness from Bayesian statistics, Phys. Rev. D 86 (2012) 125029 [arXiv: 1204.4940] [INSPIRE].

[56] M.W. Cahill-Rowley, J.L. Hewett, A. Ismail and T.G. Rizzo, The Higgs sector and fine-tuning in the pMSSM, Phys. Rev. D 86 (2012) 075015 [arXiv:1206.5800] [INSPIRE].

[57] S. Antusch, L. Calibbi, V. Maurer, M. Monaco and M. Spinrath, Naturalness of the non-universal MSSM in the light of the recent Higgs results, JHEP 01 (2013) 187 [arXiv:1207.7236] [INSPIRE].

[58] M. Perelstein and B. Shakya, XENON100 implications for naturalness in the MSSM, NMSSM and $\lambda$-SUSY, arXiv:1208.0833 [INSPIRE].

[59] H. Baer et al., Post-LHC7 fine-tuning in the mSUGRA/CMSSM model with a $125 \mathrm{GeV}$ Higgs boson, arXiv:1210.3019 [INSPIRE].

[60] M.W. Cahill-Rowley, J.L. Hewett, A. Ismail and T.G. Rizzo, More energy, more searches, but the pMSSM lives on, arXiv:1211.1981 [INSPIRE].

[61] I. Gogoladze, F. Nasir and Q. Shafi, Non-universal gaugino masses and natural supersymmetry, Int. J. Mod. Phys. A 28 (2013) 1350046 [arXiv:1212.2593] [InSPIRE]. 
[62] C. Strege et al., Global fits of the CMSSM and NUHM including the LHC Higgs discovery and new XENON100 constraints, JCAP 04 (2013) 013 [arXiv: 1212.2636] [INSPIRE].

[63] M.E. Cabrera, J.A. Casas and R.R. de Austri, The health of SUSY after the Higgs discovery and the XENON100 data, arXiv:1212.4821 [INSPIRE].

[64] K. Kowalska, L. Roszkowski and E.M. Sessolo, Two ultimate tests of constrained supersymmetry, JHEP 06 (2013) 078 [arXiv:1302.5956] [INSPIRE].

[65] G. D'Ambrosio, G. Giudice, G. Isidori and A. Strumia, Minimal flavor violation: an effective field theory approach, Nucl. Phys. B 645 (2002) 155 [hep-ph/0207036] [InSPIRE].

[66] MSSM Working Group collaboration, A. Djouadi et al., The minimal supersymmetric Standard Model: group summary report, hep-ph/9901246 [INSPIRE].

[67] C.F. Berger, J.S. Gainer, J.L. Hewett and T.G. Rizzo, Supersymmetry without prejudice, JHEP 02 (2009) 023 [arXiv:0812.0980] [InSPIRE].

[68] D.J.C. MacKay, Information theory, inference, and learning algorithms, Cambridge University Press, Cambridge U.K. (2003).

[69] CDF collaboration, CDF II exotics group public page webpage, http://www-cdf.fnal.gov/physics/exotic/exotic.html.

[70] DØ collaboration, The DØ collaboration's publications: new phenomena webpage, http://www-d0.fnal.gov/d0_publications/d0_pubs_list_runII_bytopic.html\#np.

[71] WMAP collaboration, G. Hinshaw et al., Nine-year Wilkinson Microwave Anisotropy Probe (WMAP) observations: cosmological parameter results, arXiv:1212.5226 [INSPIRE].

[72] Planck collaboration, P. Ade et al., Planck 2013 results. XVI. Cosmological parameters, arXiv: 1303.5076 [INSPIRE].

[73] Fermi-LAT collaboration, M. Ackermann et al., Fermi LAT search for dark matter in gamma-ray lines and the inclusive photon spectrum, Phys. Rev. D 86 (2012) 022002 [arXiv: 1205.2739] [INSPIRE].

[74] XENON1T collaboration, E. Aprile, The XENON1T dark matter search experiment, arXiv: 1206.6288 [INSPIRE].

[75] LUX collaboration, D. Akerib et al., The Large Underground Xenon (LUX) experiment, Nucl. Instrum. Meth. A 704 (2013) 111 [arXiv:1211.3788] [INSPIRE].

[76] M. Perelstein and C. Spethmann, A collider signature of the supersymmetric golden region, JHEP 04 (2007) 070 [hep-ph/0702038] [INSPIRE].

[77] S. Chang, P.J. Fox and N. Weiner, Naturalness and Higgs decays in the MSSM with a singlet, JHEP 08 (2006) 068 [hep-ph/0511250] [INSPIRE].

[78] L.J. Hall, D. Pinner and J.T. Ruderman, A natural SUSY Higgs near 126 GeV, JHEP 04 (2012) 131 [arXiv: 1112.2703] [INSPIRE].

[79] J. Casas, A. Lleyda and C. Muñoz, Strong constraints on the parameter space of the MSSM from charge and color breaking minima, Nucl. Phys. B 471 (1996) 3 [hep-ph/9507294] [INSPIRE].

[80] The LEP Electroweak Working Group webpage, http://lepewwg.web.cern.ch/LEPEWWG/.

[81] H.E. Haber and G.L. Kane, The search for supersymmetry: probing physics beyond the Standard Model, Phys. Rept. 117 (1985) 75 [INSPIRE]. 
[82] OPAL collaboration, G. Abbiendi et al., Search for anomalous photonic events with missing energy in $e^{+} e^{-}$collisions at $\sqrt{s}=130 \mathrm{GeV}, 136 \mathrm{GeV}$ and $183 \mathrm{GeV}$, Eur. Phys. J. C 8 (1999) 23 [hep-ex/9810021] [InSPIRE].

[83] DELPHI collaboration, J. Abdallah et al., Photon events with missing energy in $e^{+} e^{-}$ collisions at $\sqrt{s}=130$ GeV to 209 GeV, Eur. Phys. J. C 38 (2005) 395 [hep-ex/0406019] [INSPIRE].

[84] DØ collaboration, V.M. Abazov et al., Search for $Z \gamma$ events with large missing transverse energy in $p \bar{p}$ collisions at $\sqrt{s}=1.96$ TeV, Phys. Rev. D 86 (2012) 071701 [arXiv:1203.5311] [INSPIRE].

[85] CMS collaboration, Search for physics beyond the Standard Model in events with a $Z$ boson, jets and missing transverse energy in pp collisions at $\sqrt{s}=7$ TeV, Phys. Lett. $\mathbf{B} 716$ (2012) 260 [arXiv:1204.3774] [INSPIRE].

[86] T. Falk, K.A. Olive and M. Srednicki, Heavy sneutrinos as dark matter, Phys. Lett. B 339 (1994) 248 [hep-ph/9409270] [INSPIRE].

[87] T. Hebbeker, Can the sneutrino be the lightest supersymmetric particle?, Phys. Lett. B 470 (1999) 259 [hep-ph/9910326] [INSPIRE].

[88] C. Arina and N. Fornengo, Sneutrino cold dark matter, a new analysis: relic abundance and detection rates, JHEP 11 (2007) 029 [arXiv:0709.4477] [INSPIRE].

[89] C. Arina, F. Bazzocchi, N. Fornengo, J. Romao and J. Valle, Minimal supergravity sneutrino dark matter and inverse seesaw neutrino masses, Phys. Rev. Lett. 101 (2008) 161802 [arXiv:0806.3225] [INSPIRE].

[90] H. An, P.B. Dev, Y. Cai and R. Mohapatra, Sneutrino dark matter in gauged inverse seesaw models for neutrinos, Phys. Rev. Lett. 108 (2012) 081806 [arXiv:1110.1366] [INSPIRE].

[91] G. Bélanger, J. Da Silva and A. Pukhov, The right-handed sneutrino as thermal dark matter in U(1) extensions of the MSSM, JCAP 12 (2011) 014 [arXiv:1110.2414] [INSPIRE].

[92] B. Dumont, G. Bélanger, S. Fichet, S. Kraml and T. Schwetz, Mixed sneutrino dark matter in light of the 2011 XENON and LHC results, JCAP 09 (2012) 013 [arXiv:1206.1521] [INSPIRE].

[93] P. Bhupal Dev, S. Mondal, B. Mukhopadhyaya and S. Roy, Phenomenology of light sneutrino dark matter in cMSSM/mSUGRA with inverse seesaw, JHEP 09 (2012) 110 [arXiv: 1207.6542] [INSPIRE].

[94] V. De Romeri and M. Hirsch, Sneutrino dark matter in low-scale seesaw scenarios, JHEP 12 (2012) 106 [arXiv: 1209.3891] [INSPIRE].

[95] ALEPH collaboration, A. Heister et al., Search for scalar leptons in $e^{+} e^{-}$collisions at center-of-mass energies up to 209 GeV, Phys. Lett. B 526 (2002) 206 [hep-ex/0112011] [INSPIRE].

[96] ATLAS collaboration, Search for direct slepton and gaugino production in final states with two leptons and missing transverse momentum with the ATLAS detector in pp collisions at $\sqrt{s}=7$ TeV, Phys. Lett. B 718 (2013) 879 [arXiv: 1208.2884] [INSPIRE].

[97] S.S. AbdusSalam, B.C. Allanach, F. Quevedo, F. Feroz and M. Hobson, Fitting the phenomenological MSSM, Phys. Rev. D 81 (2010) 095012 [arXiv:0904.2548] [INSPIRE]. 
[98] S.S. AbdusSalam, LHC-7 supersymmetry search interpretation within the pMSSM, arXiv: 1211.0999 [INSPIRE].

[99] LHCB collaboration, Strong constraints on the rare decays $B_{s} \rightarrow \mu^{+} \mu^{-}$and $B^{0} \rightarrow \mu^{+} \mu^{-}$, Phys. Rev. Lett. 108 (2012) 231801 [arXiv:1203.4493] [INSPIRE].

[100] A. Arbey, M. Battaglia, F. Mahmoudi and D. Martinez Santos, Supersymmetry confronts $B_{s} \rightarrow \mu^{+} \mu^{-}$: present and future status, Phys. Rev. D 87 (2013) 035026 [arXiv:1212.4887] [INSPIRE].

[101] Heavy Flavor Averaging Group collaboration, D. Asner et al., Averages of b-hadron, c-hadron and $\tau$-lepton properties, arXiv:1010.1589 [INSPIRE].

[102] M. Misiak et al., Estimate of $B\left(\bar{B} \rightarrow X_{s} \gamma\right)$ at $O\left(\alpha_{s}^{2}\right)$, Phys. Rev. Lett. 98 (2007) 022002 [hep-ph/0609232] [INSPIRE].

[103] H. Baer and M. Brhlik, QCD improved $b \rightarrow s \gamma$ constraints on the minimal supergravity model, Phys. Rev. D 55 (1997) 3201 [hep-ph/9610224] [INSPIRE].

[104] K. Hagiwara, R. Liao, A.D. Martin, D. Nomura and T. Teubner, $(g-2)_{\mu}$ and $\alpha\left(M_{Z}^{2}\right)$ re-evaluated using new precise data, J. Phys. G 38 (2011) 085003 [arXiv:1105.3149] [INSPIRE].

[105] T. Moroi, The muon anomalous magnetic dipole moment in the minimal supersymmetric Standard Model, Phys. Rev. D 53 (1996) 6565 [Erratum ibid. D 56 (1997) 4424] [hep-ph/9512396] [INSPIRE].

[106] J.L. Feng and K.T. Matchev, Supersymmetry and the anomalous magnetic moment of the muon, Phys. Rev. Lett. 86 (2001) 3480 [hep-ph/0102146] [INSPIRE].

[107] U. Chattopadhyay and P. Nath, Upper limits on sparticle masses from $g-2$ and the possibility for discovery of SUSY at colliders and in dark matter searches, Phys. Rev. Lett. 86 (2001) 5854 [hep-ph/0102157] [INSPIRE].

[108] ATLAS collaboration, An update of combined measurements of the new Higgs-like boson with high mass resolution channels, ATLAS-CONF-2012-170, CERN, Geneva Switzerland (2012).

[109] CMS collaboration, Combination of Standard Model Higgs boson searches and measurements of the properties of the new boson with a mass near $125 \mathrm{GeV}$, CMS-PAS-HIG-12-045, CERN, Geneva Switzerland (2012).

[110] ATLAS collaboration, Search for the neutral Higgs bosons of the minimal supersymmetric Standard Model in pp collisions at $\sqrt{s}=7 \mathrm{TeV}$ with the ATLAS detector, JHEP 02 (2013) 095 [arXiv: 1211.6956] [INSPIRE].

[111] CMS collaboration, Search for a Higgs boson decaying into a b-quark pair and produced in association with $b$ quarks in proton-proton collisions at $7 \mathrm{TeV}$, Phys. Lett. B 722 (2013) 207 [arXiv: 1302 .2892] [INSPIRE].

[112] ATLAS collaboration, Search for charged Higgs bosons decaying via $H^{+} \rightarrow \tau \nu$ in $t \bar{t}$ events using $4.6 \mathrm{fb}^{-1}$ of pp collision data at $\sqrt{s}=7 \mathrm{TeV}$ with the ATLAS detector,

ATLAS-CONF-2012-011, CERN, Geneva Switzerland (2012).

[113] CMS collaboration, Search for a light charged Higgs boson in top quark decays in pp collisions at $\sqrt{s}=7 \mathrm{TeV}$, JHEP 07 (2012) 143 [arXiv:1205.5736] [INSPIRE]. 
[114] N.D. Christensen, T. Han and S. Su, MSSM Higgs bosons at the LHC, Phys. Rev. D 85 (2012) 115018 [arXiv: 1203.3207] [INSPIRE].

[115] M. Carena, S. Heinemeyer, O. Stål, C. Wagner and G. Weiglein, MSSM Higgs boson searches at the LHC: benchmark scenarios after the discovery of a Higgs-like particle, arXiv: 1302.7033 [INSPIRE].

[116] P.P. Giardino, K. Kannike, I. Masina, M. Raidal and A. Strumia, The universal Higgs fit, arXiv:1303.3570 [INSPIRE].

[117] L.J. Hall, K. Jedamzik, J. March-Russell and S.M. West, Freeze-in production of FIMP dark matter, JHEP 03 (2010) 080 [arXiv:0911.1120] [INSPIRE].

[118] X. Chu, T. Hambye and M.H. Tytgat, The four basic ways of creating dark matter through a portal, JCAP 05 (2012) 034 [arXiv:1112.0493] [INSPIRE].

[119] A.J. Williams, C. Boehm, S.M. West and D.A. Vasquez, Regenerating WIMPs in the light of direct and indirect detection, Phys. Rev. D 86 (2012) 055018 [arXiv:1204.3727] [INSPIRE].

[120] C. Boehm, P. Fayet and J. Silk, Light and heavy dark matter particles, Phys. Rev. D 69 (2004) 101302 [hep-ph/0311143] [INSPIRE].

[121] A. Aguirre and M. Tegmark, Multiple universes, cosmic coincidences and other dark matters, JCAP 01 (2005) 003 [hep-th/0409072] [INSPIRE].

[122] X. Chen and S.-H. Henry Tye, Heating in brane inflation and hidden dark matter, JCAP 06 (2006) 011 [hep-th/0602136] [INSPIRE].

[123] T. Hur, H.-S. Lee and S. Nasri, A supersymmetric U(1)' model with multiple dark matters, Phys. Rev. D 77 (2008) 015008 [arXiv:0710.2653] [INSPIRE].

[124] J.L. Feng and J. Kumar, The WIMPless miracle: dark-matter particles without weak-scale masses or weak interactions, Phys. Rev. Lett. 101 (2008) 231301 [arXiv:0803.4196] [INSPIRE].

[125] K.M. Zurek, Multi-component dark matter, Phys. Rev. D 79 (2009) 115002 [arXiv: 0811.4429] [INSPIRE].

[126] D. Feldman, Z. Liu, P. Nath and G. Peim, Multicomponent dark matter in supersymmetric hidden sector extensions, Phys. Rev. D 81 (2010) 095017 [arXiv:1004.0649] [INSPIRE].

[127] P.T. Winslow, K. Sigurdson and J.N. Ng, Multi-state dark matter from spherical extra dimensions, Phys. Rev. D 82 (2010) 023512 [arXiv: 1005.3013] [INSPIRE].

[128] K.R. Dienes and B. Thomas, Dynamical dark matter: I. Theoretical overview, Phys. Rev. D 85 (2012) 083523 [arXiv:1106.4546] [INSPIRE].

[129] D. Chialva, P.B. Dev and A. Mazumdar, Multiple dark matter scenarios from ubiquitous stringy throats, Phys. Rev. D 87 (2013) 063522 [arXiv:1211.0250] [INSPIRE].

[130] CMS collaboration, Search for dark matter and large extra dimensions in monojet events in pp collisions at $\sqrt{s}=7 \mathrm{TeV}$, JHEP 09 (2012) 094 [arXiv: 1206.5663] [INSPIRE].

[131] ATLAS collaboration, Search for dark matter candidates and large extra dimensions in events with a jet and missing transverse momentum with the ATLAS detector, JHEP 04 (2013) 075 [arXiv: 1210.4491] [INSPIRE].

[132] CMS collaboration, Search for dark matter and large extra dimensions in pp collisions yielding a photon and missing transverse energy, Phys. Rev. Lett. 108 (2012) 261803 [arXiv: 1204.0821] [INSPIRE]. 
[133] ATLAS collaboration, Search for dark matter candidates and large extra dimensions in events with a photon and missing transverse momentum in pp collision data at $\sqrt{s}=7 \mathrm{TeV}$ with the ATLAS detector, Phys. Rev. Lett. 110 (2013) 011802 [arXiv:1209.4625] [INSPIRE].

[134] P.J. Fox, R. Harnik, J. Kopp and Y. Tsai, LEP shines light on dark matter, Phys. Rev. D 84 (2011) 014028 [arXiv: 1103.0240] [INSPIRE].

[135] G. Hutsi, J. Chluba, A. Hektor and M. Raidal, WMAP7 and future CMB constraints on annihilating dark matter: implications on GeV-scale WIMPs, Astron. Astrophys. $\mathbf{5 3 5}$ (2011) A26 [arXiv:1103.2766] [INSPIRE].

[136] S. Galli, F. Iocco, G. Bertone and A. Melchiorri, Updated CMB constraints on dark matter annihilation cross-sections, Phys. Rev. D 84 (2011) 027302 [arXiv:1106.1528] [INSPIRE].

[137] A. Natarajan, A closer look at CMB constraints on WIMP dark matter, Phys. Rev. D 85 (2012) 083517 [arXiv:1201.3939] [INSPIRE].

[138] G. Giesen, J. Lesgourgues, B. Audren and Y. Ali-Haimoud, CMB photons shedding light on dark matter, JCAP 12 (2012) 008 [arXiv: 1209. 0247] [INSPIRE].

[139] C. Evoli, S. Pandolfi and A. Ferrara, CMB constraints on light dark matter candidates, arXiv: 1210.6845 [INSPIRE].

[140] L. Lopez-Honorez, O. Mena, S. Palomares-Ruiz and A.C. Vincent, Constraints on dark matter annihilation from CMB observations before Planck, arXiv:1303.5094 [INSPIRE].

[141] B. Henning and H. Murayama, Constraints on light dark matter from big bang nucleosynthesis, arXiv:1205.6479 [INSPIRE].

[142] Fermi-LAT collaboration, M. Ackermann et al., Constraining dark matter models from a combined analysis of milky way satellites with the Fermi Large Area Telescope, Phys. Rev. Lett. 107 (2011) 241302 [arXiv:1108.3546] [INSPIRE].

[143] R. Cotta et al., Constraints on the pMSSM from LAT observations of dwarf spheroidal galaxies, JCAP 04 (2012) 016 [arXiv:1111.2604] [inSPIRE].

[144] A. Pukhov, CalcHEP 2.3: MSSM, structure functions, event generation, batchs and generation of matrix elements for other packages, hep-ph/0412191 [INSPIRE].

[145] G. Bélanger, F. Boudjema, A. Pukhov and A. Semenov, MicrOMEGAs 2.0: a program to calculate the relic density of dark matter in a generic model, Comput. Phys. Commun. 176 (2007) 367 [hep-ph/0607059] [INSPIRE].

[146] G. Bélanger, F. Boudjema, A. Pukhov and A. Semenov, Dark matter direct detection rate in a generic model with MicrOMEGAs 2.2, Comput. Phys. Commun. 180 (2009) 747 [arXiv: 0803.2360] [INSPIRE].

[147] G. Bélanger et al., Indirect search for dark matter with MicrOMEGAs 2.4, Comput. Phys. Commun. 182 (2011) 842 [arXiv: 1004.1092] [INSPIRE].

[148] B. Allanach, SOFTSUSY: a program for calculating supersymmetric spectra, Comput. Phys. Commun. 143 (2002) 305 [hep-ph/0104145] [INSPIRE].

[149] N. Arkani-Hamed, A. Delgado and G. Giudice, The well-tempered neutralino, Nucl. Phys. B 741 (2006) 108 [hep-ph/0601041] [INSPIRE].

[150] J.F. Navarro, C.S. Frenk and S.D. White, The structure of cold dark matter halos, Astrophys. J. 462 (1996) 563 [astro-ph/9508025] [INSPIRE]. 
[151] J.F. Navarro, C.S. Frenk and S.D. White, A universal density profile from hierarchical clustering, Astrophys. J. 490 (1997) 493 [astro-ph/9611107] [INSPIRE].

[152] J. Kumar and P. Sandick, Gamma rays from bino-like dark matter in the MSSM, arXiv: 1303.2384 [INSPIRE].

[153] M. Endo, K. Hamaguchi, S. Iwamoto and T. Yoshinaga, Muon g-2 vs LHC in supersymmetric models, arXiv: 1303.4256 [INSPIRE].

[154] M. Carena, S. Gori, N.R. Shah, C.E. Wagner and L.-T. Wang, Light stau phenomenology and the Higgs $\gamma \gamma$ rate, JHEP 07 (2012) 175 [arXiv:1205.5842] [INSPIRE].

[155] T. Kitahara and T. Yoshinaga, Stau with large mass difference and enhancement of the Higgs to diphoton decay rate in the MSSM, JHEP 05 (2013) 035 [arXiv:1303.0461] [INSPIRE].

[156] M. Carena, S. Gori, N.R. Shah, C.E.M. Wagner and L.-T. Wang, Light stops, light staus and the $125 \mathrm{GeV}$ Higgs, arXiv: 1303.4414 [INSPIRE].

[157] ATLAS collaboration, Measurements of the properties of the Higgs-like boson in the two photon decay channel with the ATLAS detector using $25 \mathrm{fb}^{-1}$ of proton-proton collision data, ATLAS-CONF-2013-012, CERN, Geneva Switzerland (2013).

[158] CDMS collaboration, R. Agnese et al., Dark matter search results using the silicon detectors of CDMS II, arXiv:1304.4279 [INSPIRE]. 